\title{
The Impact of Scaling Methods on the Properties and Interpretation of Parameter Estimates in Structural Equation Models with Latent Variables
}

\author{
Eric Klopp* \\ Department of Education \\ Saarland University
}

\author{
Stefan Klößner \\ Statistics and Econometrics \\ Saarland University
}

March 7, 2021

\begin{abstract}
Latent variables in structural equation models do not have an observable scale. Hence researchers resort to scaling methods, such as fixed marker, effects coding, or fixed factor, to assign scales to the latent variables. The use of such procedures results in numerically different estimates, in spite of a single underlying population model. In this paper, we provide a framework which not only allows for a translation between estimates obtained under different scaling methods, but also helps to explore the relation between the underlying population parameters and their estimates, thus providing a basis for the interpretation of estimated parameters. Additionally, the framework proves useful for demonstrating that the choice of scaling method affects the power of the Wald test for testing parameters' significance.
\end{abstract}

Keywords: scaling of latent variables, parameter estimates, parameter conversion, interpretation of parameter estimates

This article has been published in Structural Equation Modeling: A Multidisciplinary Journal on March 1, 2021. Please use the following citation:

Klopp, E. \& Klößner, S. (2021). The Impact of the Choice of Scaling Method on the Properties and Interpretation of Parameter Estimates in Structural Equation Models with Latent Variables. Structural Equation Modeling: A Multidisciplinary Journal, doi: 10.1080/10705511.2020.1796673.

${ }^{*}$ Corresponding author: Eric Klopp, Saarland University, Department of Education, Bldg. A 4.2, 66123 Saarbrücken, Germany, email: e.klopp@mx.uni-saarland.de. 


\section{Introduction}

One of the main goals in introductory statistic classes in regression analysis is to familiarize students with the interpretation of the regression coefficient. For instance, consider the manifest regression model depicted in Figure 1, which is a rather simple structural equation model $(\mathrm{SEM})$. If the exogenous variable $\xi_{1}$ is continuous, the parameter of interest is the regression coefficient that indicates the relation between the exogenous variable $\xi_{1}$ and the endogenous variable $\eta_{1}$. The interpretation of the unstandardized regression coefficient or, in terms of SEM, the structural parameter $\gamma_{11}$ is that a one unit increase of $\xi_{1}$ yields a 3 unit increase of $\eta_{1}$. When drawing a sample from this population, the estimated regression coefficient would approximately equal the population value, with small fluctuations resulting from sampling error. We now consider the case in which both the exogenous variable $\xi_{1}$ and the endogenous variable $\eta_{1}$ are latent variables. The graph of the corresponding SEM is depicted in Figure 2. In contrast to a SEM with manifest variables only, in a SEM involving latent variables the latter need to be scaled. Otherwise, the model is not be identified and parameters cannot be uniquely estimated. A common scaling method, which is the de facto standard in most SEM software, is the Fixed Marker (FM) scaling (cf. Kline, 2016, pp. 199-200). In the FM method, one of the factor loadings per latent variable is restricted to 1 and the corresponding manifest variable is called the referent indicator. In SEM, it is an at least implicitly known fact that when latent variables are involved, the results of the estimation process depend on the scaling method used to scale the latent variables, i.e., the estimated parameters are relative to the choice of the scaling method. Consequently, the interpretation of estimated parameters also depends on the choice of the scaling method. The issue of the interpretation of parameter estimates in SEMs involving latent variables has been recently discussed by Klößner and Klopp (2019) in an explanation of the phenomenon of constraint interaction (Steiger, 2002). Klößner and Klopp demonstrated the dependency of the estimated parameters on the scaling method. Estimated parameters represent an algebraic transformation of population parameters. Hence, the question arises how the regression coefficient should be interpreted when the estimated coefficient reflects an algebraic transformation of specific population parameters. However, these authors did not cover regression coefficients.

An attempt to answer this question was undertaken by Bielby (1986) who discussed the effects of the latent variables' scaling on the estimated regression coefficient in a SEM akin to the one given in Figure 2. Using FM scaling, Bielby demonstrated that in addition to the fluctuations due to sampling error, the estimated regression coefficient systematically 
differs from the population regression coefficient if the population loading ${ }^{1}$ of the restricted parameter differs numerically from 1 , the value to which it is fixed as scaling constraint. Drawing on the example of Bielby (1986), we will now demonstrate this issue using the model from Figure 2. The population values for the model parameters are indicated in this figure. To estimate this model, we will use all three common scaling methods. Besides the Fixed Marker (FM) method, these are the Effects Coding (EC) method and the Fixed Factor (FF) method (cf. Kline, 2016, pp. 199-200). ${ }^{2}$ Now, we estimate the model from Figure 2 with a sample of size $N=500$ drawn from the population depicted in the figure for the corresponding scaling methods ${ }^{3}$. Irrespective of the scaling method employed, the model fit the data well, $\chi^{2}(1)=1.472, p=.225, \mathrm{CFI}=1.000, \mathrm{SRMR}=0.002, \mathrm{RMSEA}=0.031$, Table 1 provides the results for estimated parameters: we find that neither of the estimated regression coefficients $\widehat{\gamma}_{11}$ under the three scaling methods equals its population equivalent, replicating the result of Bielby (1986). Consequently, on the one hand, the example shows that, given the knowledge of the population regression coefficient in this hypothetical example, the estimated regression cannot be interpreted in the same way as in the regression model with manifest variables only. On the other hand, we show that the problem mentioned by Bielby (1986) also occurs in the context of EC and FF scaling, and, drawing on results provided in Klößner and Klopp (2019), the question arises which algebraic transformation of population parameters the estimated regression coefficient represents under the different scaling methods.

Besides the question of how to interpret the estimated parameters, there is another issue, which is occasionally mentioned in the literature, and refers to the possibility to convert the estimated parameters obtained under a given scaling method to the estimated parameters without the need to re-estimate the model. Newsom (2015, pp. 4-5) provided formulas to convert the estimated loadings and variances of the latent variable between the FM and FF scaling method (see also Skrondal \& Rabe-Hesketh, 2004, chapter 5.3.2). This conversion of the estimated factor loadings simply requires the multiplication of the estimated loading with the estimated standard deviation under the FM scaling to get the estimated loading under the FF scaling. For instance, using the estimated loadings for the exogenous latent variable from Table 1, we can calculate the estimated loading $\widehat{\lambda}_{x, 11}$ under the FF scaling as $\widehat{\lambda}_{x, 11}=1.000 \cdot \sqrt{0.953}=0.976$, which is exactly the parameter's value from Table 1 . The

\footnotetext{
${ }^{1}$ Bielby (1986) used the term "true parameter", in this paper we use the term "population parameter" instead.

${ }^{2}$ While FM scaling fixes one arbitrarily chosen estimated loading to unity (usually the first indicator of a latent variable), EC scaling forces the estimated loadings' average to unity, and the FF method fixes to unity the exogenous latent variables' variances as well as the endogenous latent variables' residual variances.

${ }^{3}$ The covariances matrices for all examples in this paper are given in the supplementary material. In all examples in this contribution, we use simulated data. All calculations were done with R (R Core Team, 2018) and the lavaan package (Rosseel, 2012) in version 0.6.3.
} 
same holds for the loading of the second indicator of the exogenous latent variable and also straightforwardly applies to the loadings of the endogenous latent variable. From this example, the question arises if it is possible to convert the other parameters in a SEM like regression coefficients and if such conversions are also possible for all conceivable combinations of the three scaling methods. After all, there is the question why such conversions are possible.

With regard to different estimates yielded by different scaling methods, there are some contributions that demonstrate the dependence of the Wald test for single parameters on the applied scaling method (de Pijper \& Saris, 1982; Gonzalez \& Griffin, 2001). This raises the question whether the different behavior of the Wald test under different scaling methods can be described systematically. From a practical perspective, there is the question if there are differences in the power of the Wald tests for the same parameters estimated under different scaling methods.

The goal of this paper is to shed light on the questions put forward in this section. To reach this goal, we provide the mathematical foundation of scaling methods in the second section and derive and prove a proposition regarding the conversion of estimated parameters as well as a proposition about the relation between population values and their estimates. We will show that the relation between estimated and population parameters and the conversion of estimated parameters between two different scaling methods share the same mathematical foundation, which is one of the first main contributions of this paper. Moreover, we will provide a formal account on the topic of scaling methods and the consequences thereof. To the best of our knowledge, this is the first attempt to provide such an account. In the third section, we apply the results from the second section to the initial regression model. In particular, we demonstrate the formulas for parameter conversion from the second section in practice and explain the interpretation of the estimated parameters using the regression examples, which is the second main contribution of this paper. In addition, we elaborate on the issues of interpretation and parameter conversion for misspecified models. In the forth section, we use a more complex model - a mediation model - and investigate the behavior of the Wald test for single parameters using the results from the second section. Thus, the fourth section is an application of the methods developed in the second section and also demonstrates a consequence of the need to scale a SEM for the Wald test. The fifth and last section provides some conclusions and remarks.

\section{Mathematical Derivation}

This section provides the paper's mathematical foundations. After introducing the general setup and necessary definitions in subsection 2.1 , we provide results on how to convert es- 
timated parameters (and transformations thereof) from one scaling method to another in subsection 2.2 , without having to re-estimate the model. In subsection 2.3, we show how estimated parameters relate to population parameters, providing the foundation for answering the question of how to interpret estimated parameters. Finally, subsection 2.4 recaps the mathematical foundation and summarizes how this framework is applied in the preceding sections.

Before we introduce the general mathematical setup, we want to highlight a restriction we made to ease our considerations. Firstly, we only consider the scalings used in SEMs with the common confirmatory factor analysis style measurement model. Secondly, we do not consider the application of scaling restrictions in the kind of, e.g., latent growth curve models (see Newsom, 2015), or the use of scaling restrictions to investigate item position effects (e.g., Schweizer, 2011). The following sections are written in a mathematical style, i.e., we provide our main assertions in the form of propositions, which are also proven. Some lemmata and proofs thereof, that are not directly necessary to the main propositions, are put in the mathematical appendix. Some other important issues are mentioned in remarks, for ease of later referral.

\subsection{Setup}

Regarding notation, we employ the full LISREL notation. However, as we do not consider manifest and latent variables' intercepts, we omit corresponding intercept terms. In the full LISREL notation, the measurement model for the exogenous variables is given by

$$
\boldsymbol{X}=\boldsymbol{\Lambda}_{x} \boldsymbol{\xi}+\boldsymbol{\delta}
$$

where the $q$-dimensional vector $\boldsymbol{X}$ consists of the manifest variables measuring the exogenous latent variables contained in the $n$-dimensional vector $\boldsymbol{\xi}, \boldsymbol{\Lambda}_{x}$ is a $q \times n$ matrix of factor loadings, and $\boldsymbol{\delta}$ is a $q$-dimensional vector of measurement errors. Analogously, the measurement model for the endogenous variables is given by

$$
\boldsymbol{Y}=\boldsymbol{\Lambda}_{y} \boldsymbol{\eta}+\boldsymbol{\epsilon}
$$

where the $p$-dimensional vector $\boldsymbol{Y}$ consists of the manifest variables measuring the endogenous latent variables contained in the $m$-dimensional vector $\boldsymbol{\eta}, \boldsymbol{\Lambda}_{y}$ is a $p \times m$ matrix of factor loadings, and $\boldsymbol{\epsilon}$ is a $p$-dimensional vector of measurement errors. Finally, the structural model is given by

$$
\eta=B \eta+\Gamma \xi+\zeta
$$


where $\boldsymbol{B}$ is an $m \times m$ matrix containing the effects of the endogenous variables on themselves, $\boldsymbol{\Gamma}$ is an $m \times n$ matrix consisting of the exogenous variables' direct effects on the endogenous variables, and $\boldsymbol{\zeta}$ is an $m$-dimensional error term.

Under the standard assumptions that $\boldsymbol{\delta}, \boldsymbol{\epsilon}, \boldsymbol{\zeta}, \boldsymbol{\xi}$ are all mutually uncorrelated and that $\boldsymbol{A}:=(\boldsymbol{I}-\boldsymbol{B})^{-1}$ exists $^{4}$ (Bollen, 1989, p. 14-20), the covariance matrix $\boldsymbol{\Sigma}$ of the vector $\boldsymbol{Z}:=\left(\begin{array}{l}\boldsymbol{Y} \\ \boldsymbol{X}\end{array}\right)$ of manifest variables can be deduced (Bollen, 1989, p. 319-326):

$$
\boldsymbol{\Sigma}(\theta)=\left(\begin{array}{cc}
\boldsymbol{\Lambda}_{y} \boldsymbol{A}\left(\boldsymbol{\Gamma} \boldsymbol{\Phi} \boldsymbol{\Gamma}^{\top}+\boldsymbol{\Psi}\right) \boldsymbol{A}^{\top} \boldsymbol{\Lambda}_{y}^{\top}+\boldsymbol{\Theta}_{\epsilon} & \boldsymbol{\Lambda}_{y} \boldsymbol{A} \boldsymbol{\Gamma} \boldsymbol{\Phi} \boldsymbol{\Lambda}_{x}^{\top} \\
\boldsymbol{\Lambda}_{x} \boldsymbol{\Phi} \boldsymbol{\Gamma}^{\top} \boldsymbol{A}^{\top} \boldsymbol{\Lambda}_{y}^{\top} & \boldsymbol{\Lambda}_{x} \boldsymbol{\Phi} \boldsymbol{\Lambda}_{x}^{\top}+\boldsymbol{\Theta}_{\delta}
\end{array}\right)
$$

where $\boldsymbol{\Phi}$ and $\boldsymbol{\Psi}$ denote the covariance matrices of $\boldsymbol{\xi}$ and $\boldsymbol{\zeta}$, and $\boldsymbol{\Theta}_{\delta}$ and $\boldsymbol{\Theta}_{\epsilon}$ are diagonal matrices denoting the variances of $\boldsymbol{\delta}$ and $\boldsymbol{\epsilon}$, while the 8-tuple

$$
\theta:=\left(\boldsymbol{\Lambda}_{x}, \boldsymbol{\Lambda}_{y}, \boldsymbol{\Gamma}, \boldsymbol{B}, \boldsymbol{\Phi}, \boldsymbol{\Psi}, \boldsymbol{\Theta}_{\delta}, \boldsymbol{\Theta}_{\epsilon}\right)
$$

collects the parameters of the structural equation model.

As latent variables are not observable per se, their scale is not observable, either. In the following thought experiment, though, we suppose that we are considering exogenous latent variables $\boldsymbol{\xi}$ whose scale we know: these exogenous latent variables are assumed to reflect amounts of money, measured in units of the corresponding currency. Suppose now that a new currency is introduced. ${ }^{5}$ Suppose further that one unit of the new currency corresponds to 1,000 units of the old currency. Consequently, the scale of these exogenous latent variables $\boldsymbol{\xi}$ changes. Now, instead of $\boldsymbol{\xi}$, the corresponding values would be given by $\widetilde{\boldsymbol{\xi}}:=\frac{1}{1000} \cdot \boldsymbol{\xi}$. Due to the well-known formula $\operatorname{Var}(c \boldsymbol{\xi})=c^{2} \operatorname{Var}(\boldsymbol{\xi})$ for any constant $c$, the covariance matrix of $\widetilde{\boldsymbol{\xi}}$ would be $\widetilde{\boldsymbol{\Phi}}:=\frac{1}{1000^{2}} \cdot \boldsymbol{\Phi}$, and with respect to $\widetilde{\boldsymbol{\xi}}$, the loading matrix would be $\widetilde{\boldsymbol{\Lambda}}_{x}:=1000 \cdot \boldsymbol{\Lambda}_{x}$, while the effects of the latent exogenous variables $\widetilde{\boldsymbol{\xi}}$ on the latent endogenous variables would be described by $\widetilde{\boldsymbol{\Gamma}}:=1000 \cdot \boldsymbol{\Gamma}$. Inspection of Equation (4) shows that the manifest variables' covariance matrix would not change at all if we replaced $\boldsymbol{\Lambda}_{x}, \boldsymbol{\Gamma}$, and $\boldsymbol{\Phi}$ by $\widetilde{\boldsymbol{\Lambda}}, \widetilde{\boldsymbol{\Gamma}}$, and $\widetilde{\boldsymbol{\Phi}}$, respectively.

These considerations can be generalized by changing the scale of each component of $\boldsymbol{\xi}$ individually, using a diagonal matrix $\boldsymbol{D}_{\xi}$ whose diagonal contains the respective entries, $d_{\xi, 1}, \ldots, d_{\xi, n} \neq 0$. One would then replace $\boldsymbol{\xi}, \boldsymbol{\Lambda}_{x}, \boldsymbol{\Gamma}$, and $\boldsymbol{\Phi}$ by $\widetilde{\boldsymbol{\xi}}:=\boldsymbol{D}_{\xi}^{-1} \boldsymbol{\xi}, \widetilde{\boldsymbol{\Lambda}}_{x}:=\boldsymbol{\Lambda}_{x} \boldsymbol{D}_{\xi}$, $\widetilde{\boldsymbol{\Gamma}}:=\boldsymbol{\Gamma} \boldsymbol{D}_{\xi}$, and $\widetilde{\boldsymbol{\Phi}}:=\boldsymbol{D}_{\xi}^{-1} \boldsymbol{\Phi} \boldsymbol{D}_{\xi}^{-1}$, respectively. Again, such a change would not affect the manifest variables' covariance matrix. Similarly, one might also change the scales of the latent en-

\footnotetext{
${ }^{4}$ As it is common in mathematics, we use the symbol ' $:=$ ' to indicate that the corresponding left-hand side variable is defined as the corresponding right-hand side term.

${ }^{5} \mathrm{An}$ example is the introduction of the Euro in some countries of the European Union. For instance, 1 Euro corresponds to 1.95583 Deutschmark, the former German currency.
} 
dogenous variables, $\boldsymbol{\eta}$, by using a diagonal matrix $\boldsymbol{D}_{\eta}$ whose diagonal entries $d_{\eta, 1}, \ldots, d_{\eta, m} \neq 0$ are used for rescaling $\boldsymbol{\eta}$ to $\tilde{\boldsymbol{\eta}}:=\boldsymbol{D}_{\eta}^{-1} \boldsymbol{\eta}$. Taking these ideas together, we arrive at the following definition which considers changes of the latent variables' scales. ${ }^{6}$

Definition 1 (Change of Scale). For diagonal matrices $\boldsymbol{D}_{\xi} \in \mathbb{R}^{n \times n}$ and $\boldsymbol{D}_{\eta} \in \mathbb{R}^{m \times m}$ with non-zero entries ${ }^{7}$ :

1. We say that $\widetilde{\theta}:=\left(\widetilde{\boldsymbol{\Lambda}}_{x}, \widetilde{\boldsymbol{\Lambda}}, \widetilde{\boldsymbol{\Gamma}}, \widetilde{\boldsymbol{B}}, \widetilde{\boldsymbol{\Phi}}, \widetilde{\boldsymbol{\Psi}}, \widetilde{\boldsymbol{\Theta}}_{\delta}, \widetilde{\boldsymbol{\Theta}}_{\epsilon}\right)$ is derived from $\theta$ via a change of scale according to $\boldsymbol{D}_{\xi}, \boldsymbol{D}_{\eta}$ if

$$
\begin{aligned}
\widetilde{\boldsymbol{\Lambda}}_{x}=\boldsymbol{\Lambda}_{x} \boldsymbol{D}_{\xi}, \quad \widetilde{\boldsymbol{\Lambda}}_{y}=\boldsymbol{\Lambda}_{y} \boldsymbol{D}_{\eta}, \quad \widetilde{\boldsymbol{\Gamma}}=\boldsymbol{D}_{\eta}^{-1} \boldsymbol{\Gamma} \boldsymbol{D}_{\xi}, \quad \widetilde{\boldsymbol{B}}=\boldsymbol{D}_{\eta}^{-1} \boldsymbol{B} \boldsymbol{D}_{\eta} \\
\\
\widetilde{\boldsymbol{\Phi}}=\boldsymbol{D}_{\xi}^{-1} \boldsymbol{\Phi} \boldsymbol{D}_{\xi}^{-1}, \quad \widetilde{\boldsymbol{\Psi}}=\boldsymbol{D}_{\eta}^{-1} \boldsymbol{\Psi} \boldsymbol{D}_{\eta}^{-1}, \quad \widetilde{\boldsymbol{\Theta}}_{\delta}=\boldsymbol{\Theta}_{\delta}, \quad \widetilde{\boldsymbol{\Theta}}_{\epsilon}=\boldsymbol{\Theta}_{\epsilon} .
\end{aligned}
$$

We write $\theta \stackrel{\boldsymbol{D}_{\xi}, \boldsymbol{D}_{\eta}}{\longrightarrow} \widetilde{\theta}$ to indicate that $\widetilde{\theta}$ is derived from $\theta$ via a change of scale according to the diagonal matrices $\boldsymbol{D}_{\xi}, \boldsymbol{D}_{\eta}$.

2. As $\theta \stackrel{\boldsymbol{D}_{\xi}, \boldsymbol{D}_{\eta}}{\sim} \widetilde{\theta}$ is equivalent to $\widetilde{\theta} \stackrel{\widetilde{\boldsymbol{D}}_{\xi}, \widetilde{\boldsymbol{D}}_{\eta}}{\sim} \theta$ with $\widetilde{\boldsymbol{D}}_{\xi}:=\boldsymbol{D}_{\xi}^{-1}$ and $\widetilde{\boldsymbol{D}}_{\eta}:=\boldsymbol{D}_{\eta}^{-1}$, we also write $\theta \leadsto \widetilde{\theta}$ and say that $\theta$ and $\widetilde{\theta}$ are related via a change of scale. ${ }^{8}$

Remark 1 (Manifest residual errors). We would like to draw the reader's attention to the fact that the manifest variables' covariance matrices, $\boldsymbol{\Theta}_{\delta}$ and $\boldsymbol{\Theta}_{\epsilon}$, stay unchanged when a change of scale is applied to the latent variables. The reason for this is that the measurement errors $\boldsymbol{\delta}$ and $\boldsymbol{\epsilon}$ are unaffected by the latent variables' scales. In contrast, all other model matrices are influenced by a change of scale, as all the entries in $\boldsymbol{\Gamma}, \boldsymbol{B}, \boldsymbol{\Phi}, \boldsymbol{\Psi}, \boldsymbol{\Lambda}_{x}$, and $\boldsymbol{\Lambda}_{y}$ constitute quantities which describe how latent variables influence other variables or how latent variables are influenced by other variables. As a rule of thumb, when looking at a graphical model description like the ones given in Figures 2 and 5, the model parameters affected by a change of scale are those for which the corresponding arrow starts and/or ends in a latent variable.

We now state the key property of change of scale.

Proposition 1. If $\theta \leadsto \widetilde{\theta}$, i.e. if $\theta$ and $\widetilde{\theta}$ are related via a change of scale, we have $\boldsymbol{\Sigma}(\theta)=$ $\Sigma(\widetilde{\theta})$.

\footnotetext{
${ }^{6}$ The considerations presented so far represent a systematization and extension of ideas that have been discussed in the SEM literature, e.g., Jöreskog (1978); Klößner and Klopp (2019); Mulaik (2010, p. 443); SanMartin, Gonzalez, and Tuerlinckx (2015); Schweizer (2011); Schweizer, Troche, and DiStefano (2019); Schweizer and Troche (2019); von Oertzen (2010); Yoon and Millsap (2007).

${ }^{7}$ As is common in mathematics, $\mathbb{R}^{n \times n}$ and $\mathbb{R}^{m \times m}$ denote the sets of $n \times n$ and $m \times m$-matrices, respectively.

${ }^{8}$ It is easy to see that $\leadsto$ defines an equivalence relation on the set of all parameters, i.e., a reflexive, symmetric, and transitive relation.
} 
Proof. The proof follows from assertions '6.' - '8.' of Lemma 2 in the appendix.

Using the new terminology, Definition 1 and Proposition 1 can be interpreted as stating that changes of the latent variables' scales do not alter the manifest variables' covariance matrix. This fact, which originates from the latent variables not being observable, hinders the structural model in its raw and unscaled form from being identified: different parameter tuples $\theta$ and $\tilde{\theta}$ can produce identical $\Sigma(\theta)=\Sigma(\widetilde{\theta})$, making it impossible to determine $\theta$ from observing the manifest variables, as $\theta$ and $\widetilde{\theta}$ imply the same distribution for the manifest variables. For the remainder of this paper, it is essential to point out that we differentiate between a raw, unscaled structural equation model and the models that are derived from it by adding scaling restrictions in order to arrive at an identified model. The original, raw model, to which we will refer as the "unscaled model", is the root of several different models: for instance, when using the Fixed Marker method (FM), the "FM-scaled model" consists of the raw, "unscaled" model in connection with the restrictions that any marker variable's loading on the corresponding latent variable equals unity. The difference between an "unscaled" and "scaled" model using the FM scaling method is depicted in Figure 3, where the scaling restrictions are shown in red. Analogously, building on the "unscaled model" and using Fixed Factor (FF) scaling, the condition that all exogenous latent variables and all error terms of endogenous latent variables shall have unit variance is added to the raw, "unscaled" model. As this paper aims at studying the relations between different scaling methods, unless stated otherwise, all our considerations are concerned with the "unscaled" model, as it is the basis of all the "scaled" models that are derived from it by adding scaling restrictions. With respect to "unscaled" models, we introduce a new definition which will prove central for parameter interpretation later on.

Definition 2 (Identified up to scaling). We call an "unscaled" structural equation model identified up to scaling if and only if it has the following property: for all $\theta$ and $\widetilde{\theta}$ with $\boldsymbol{\Sigma}(\theta)=\boldsymbol{\Sigma}(\widetilde{\theta}), \theta$ and $\widetilde{\theta}$ are related via a change of scale. Formally: $\boldsymbol{\Sigma}(\theta)=\boldsymbol{\Sigma}(\widetilde{\theta}) \Rightarrow \theta \leadsto \widetilde{\theta}$.

When a raw, "unscaled" model is identified up to scaling, then all the non-uniqueness concerning parameters is due to the unknown scales of the latent variables, as Definition 2 is obviously equivalent to $\boldsymbol{\Sigma}(\theta) \neq \boldsymbol{\Sigma}(\widetilde{\theta})$ for all $\theta$ and $\widetilde{\theta}$ that are not related by some change of scale. The definition might therefore be rephrased as stating that all non-uniqueness and identification problems of the "unscaled" SEM model stem from the fact that latent variables' scales might be changed without altering the manifest variables' distribution. This entails that an up to scaling identified model only lacks scaling restrictions to be identified. Put differently, after an up to scaling identified model has been scaled by introducing corresponding restrictions on parameters, it will be identified. 
On the other hand, models exist which are not identified up to scaling. For instance, a non-recursive model is not identified up to scaling, cf., Kline (2016, p. 135), Rigdon (1995), and Shipley (2004, p. 171-173). ${ }^{9}$ Exploratory factor models are also not identified up to scaling. Another instance of a model that is not identified up to scaling is a model with one latent variable and two indicators. These models have in common that they are not identified even after one of the usual scaling restrictions has been added to the model. However, a model with two latent variables each having two or more indicators would be identified. Thus, the unscaled regression model in Figure 3 is identified up to scaling. In exactly the same way, a model with one latent variable with at least three indicators is identified up to scaling, as applying scaling restrictions renders such a model identified (see Kline, 2016, p. 201) ${ }^{10}$. In order to determine if a model is identified up to scaling, rules exist that indicate if a model is identified or not after applying a scaling method (e.g., Shipley, 2004, p. 165).

Before we formally define the three common scaling methods, we make, for the sake of simplicity and to facilitate the upcoming considerations, the following two assumptions:

Assumption 1. For the scaled model to be identified, all latent variables need to be scaled.

Assumption 2. All latent variables are scaled by means of the same scaling method.

Assumption 1 holds true if no non-standard restrictions appear in the model matrices affected by changes of scale, i.e. in $\boldsymbol{\Gamma}, \boldsymbol{B}, \boldsymbol{\Phi}, \boldsymbol{\Psi}, \boldsymbol{\Lambda}_{x}$, and $\boldsymbol{\Lambda}_{y}$. Standard restrictions consist of fixing parameters to zero, corresponding to the absence of an effect, loading, or covariance, while non-standard restrictions are for instance equality restrictions between certain parameters, e.g. $\gamma_{11}=\gamma_{12}$, or fixing a parameter to a non-zero value, e.g. $\phi_{12}=2$. Assumption 2 is simply made to ease the following considerations. Mathematically, it is not necessary for each latent variable to be scaled by means of the same scaling method, we will discuss this assumption in the conclusion.

Now, we formally define the three common scaling methods already mentioned in the introduction (FM, EC, and FF scaling, see Kline, 2016, pp. 199-200; Little, Slegers, \& Card, 2006). To the best of our knowledge, these three common scaling methods have not been formally defined before, so we contribute to the literature by providing their formal definitions. Although usually not stated explicitly, the scaling of a structural equation model is not concerned with restrictions on population parameters, but with restrictions on the estimated values of the parameters. We therefore denote scaling restrictions in terms of estimated

\footnotetext{
${ }^{9}$ For the sake of completeness, we want to note that there may be models that are not recursive but that are nevertheless identified up to scaling, see Kline (2016, p. 135), Rigdon (1995), and Shipley (2004, p. 171-173), for the respective identification conditions.

${ }^{10}$ However, the estimated model has zero degrees of freedom so that no model evaluation is possible.
} 
parameters which we indicate by 'hats': thus, scaling restrictions refer to the estimated model parameters $\widehat{\theta}$ in general or to $\widehat{\boldsymbol{\Lambda}}_{x}, \widehat{\boldsymbol{\Lambda}}_{y}, \widehat{\boldsymbol{\Phi}}$, or $\widehat{\boldsymbol{\Psi}}$ in particular. Notice that the matrices $\boldsymbol{\Theta}_{\delta}$ and $\boldsymbol{\Theta}_{\epsilon}$ are allowed to contain any kind of restrictions, as these have no effect at all on the latent variables' scales, as explained in Remark 1 above. In particular, researchers may specify restrictions on measurement error variances in order to improve the latent variables' meaning, as advocated by Hayduk and Littvay (2012, see also Hayduk, 1988, pp. 118-125).

With regard to the restrictions they impose, all scaling methods have in common that they enforce a set of $n+m$ restrictions: one for each of the $n$ latent exogenous variables that $\boldsymbol{\xi}$ consists of, and also one for each of the $m$ latent endogenous variables collected in $\boldsymbol{\eta}$.

Definition 3 (Fixed marker scaling). For a latent variable, fixed marker scaling fixes an arbitrary estimated factor loading to unity.

1. For the $i$-th exogenous latent variable $(i \in\{1, \ldots, n\})$, a so-called referent indicator $R_{\xi, i} \in\{1, \ldots, q\}$ is chosen and for the corresponding loading, $\widehat{\lambda}_{x, R_{\xi, i}, i}=1$ is postulated.

2. For the $j$-th endogenous latent variable $(j \in\{1, \ldots, m\})$, a so-called referent indicator $R_{\eta, j} \in\{1, \ldots, p\}$ is chosen and for the corresponding loading, $\widehat{\lambda}_{y, R_{\eta, j}, j}=1$ is postulated.

For scaling a latent variable, fixed marker scaling consists of choosing a referent indicator whose loading on that latent variable is fixed to unity. Mathematically, in the corresponding column of $\widehat{\boldsymbol{\Lambda}}_{x}$ or $\widehat{\Lambda}_{y}$, one specific element must be equal to 1 . As a consequence, if all latent variables are scaled via fixed marker scaling, then every column of the loading matrices $\widehat{\Lambda}_{x}$ or $\widehat{\Lambda}_{y}$ contains one specific value which is fixed to unity. Effects coding, in contrast, does in general not fix specific parameters to unity or some other value, but employs restrictions that involve all the loadings related to a specific latent variable. In particular, effects coding fixes the average of any latent variable's non-zero factor loadings, $\mathrm{A}\left(\widehat{\lambda}_{x, \bullet i}\right)$ or $\mathrm{A}\left(\widehat{\lambda}_{y, \bullet j}\right)$, respectively, to unity.

Definition 4 (Effects coding scaling). For a latent variable, effects coding enforces that the average of its estimated factor loadings is fixed to unity ${ }^{11}$.

1. For the $i$-th exogenous latent variable $(i \in\{1, \ldots, n\}), \mathrm{A}\left(\widehat{\lambda}_{x, \bullet}\right)=1$ is postulated for the average $\mathrm{A}\left(\widehat{\lambda}_{x, \bullet i}\right):=\frac{\sum_{k=1}^{q} \widehat{\lambda}_{x, k i}}{\#\left\{k: \widehat{\lambda}_{x, k i} \neq 0\right\}}$.

\footnotetext{
${ }^{11}$ As is common in mathematics, the symbol "\#" denotes the cardinality of a set, i.e., the number of elements in the set. In Definition 4, it indicates the number of indicators for which estimated loadings are different from zero.
} 
2. For the $j$-th endogenous latent variable $(j \in\{1, \ldots, m\}), \mathrm{A}\left(\widehat{\lambda}_{y, \bullet j}\right)=1$ is postulated for the average $\mathrm{A}\left(\widehat{\lambda}_{y, \bullet j}\right):=\frac{\sum_{l=1}^{p} \widehat{\lambda}_{y, l j}}{\#\left\{l: \widehat{\lambda}_{y, l j} \neq 0\right\}}$.

The effects coding method enforces that the non-zero entries in the corresponding columns of $\widehat{\Lambda}_{x}$ or $\widehat{\Lambda}_{y}$ average to unity. As a consequence, if all latent variables are scaled using effects coding, then the non-zero entries of all the loading matrices' columns average to unity. The fixed factor method, in contrast, does not operate on loadings, but on (residual) variances related to the latent variables.

Definition 5 (Fixed factor scaling). For a latent variable, fixed factor scaling fixes its variance to unity in case of an exogenous variable, while for endogenous latent variables, their error term's variance is fixed to unity.

1. For the $i$-th exogenous latent variable $(i \in\{1, \ldots, n\})$, this variable's variance is fixed to unity: $\widehat{\phi}_{i i}=1$.

2. For the $j$-th endogenous latent variable $(j \in\{1, \ldots, m\})$, this variable's residual variance is fixed to unity: $\widehat{\psi}_{j j}=1$.

Fixed factor scaling enforces that corresponding values on the diagonals of $\widehat{\boldsymbol{\Phi}}$ and $\widehat{\Psi}$ equal unity. As a consequence, if all latent variables are scaled via fixed factor scaling, then the estimated covariance matrix of the exogenous latent variables, $\widehat{\boldsymbol{\Phi}}$, is at the same time a correlation matrix. Similarly, the estimated covariance matrix $\widehat{\Psi}$, the covariance matrix of the latent error term $\widehat{\boldsymbol{\zeta}}$, is at the same time a correlation matrix.

Remark 2 (Application to other scaling methods). The aforementioned scaling methods are the ones most commonly used, but they do not represent all possible scaling methods. For instance, Schweizer and Troche (2019) introduced the EV scaling method (eigen value scaling, because the estimated variances are close to eigen values; Schweizer \& Troche, 2019, pp. 178). The EV scaling method enforces that in every column of $\widehat{\boldsymbol{\Lambda}}_{x}$ and $\widehat{\boldsymbol{\Lambda}}_{y}$, the sum of the squared estimated loadings equals 1 . Thus, for an exogenous latent variable $\xi_{i}$, this scaling requires that $\sum_{k=1}^{q} \widehat{\lambda}_{x, k i}^{2}=1$. Accordingly, the restriction for a latent endogenous variable $\eta_{j}$ is $\sum_{l=1}^{p} \widehat{\lambda}_{y, l j}^{2}=$ $1 .^{12}$

Moreover, there is theoretically an infinite number of scaling methods, but for up to scaling identified models, the estimates obtained using different scaling methods will be related to

\footnotetext{
${ }^{12}$ In Schweizer and Troche (2019, pp. 178-179), two methods are discussed for calculating EV scaled parameters, both of which are equivalent to the condition we state in the main text.
} 
each other by a change of scale, due to the definition of "identified up to scaling" as given in Definition 2.

Remark 3 (Notation). Due to Assumption 2, all latent variables are being scaled using the same scaling method, we will therefore denote estimates derived by using fixed marker (effects coding, fixed factor, resp.) scaling for all latent variables by $\widehat{\theta}^{(\mathrm{FM})}\left(\widehat{\theta}^{(\mathrm{EC})}, \widehat{\theta}^{(\mathrm{FF})}\right.$, resp.).

Remark 4 (Fixed marker scaling). From a theoretical perspective, FM scaling splits up into various versions, depending on which indicator is used as referent indicator. For instance, in the regression model in Figure 2 and under the assumption that for each latent variable the same referent indicator is used, there are two versions of FM scaling: the first version uses the first indicator for each latent variable's scaling and, accordingly, the second version uses the second indicator for each latent variable's scaling. Thus, when necessary, we will also differentiate between $\widehat{\theta}^{\text {(FM1) }}$ and $\widehat{\theta}^{\text {(FM2) }}$, with $\widehat{\theta}^{\text {(FM1) }}$ denoting the estimates when using the factors' first indicators as referent indicators, while $\widehat{\theta}^{(\mathrm{FM} 2)}$ denotes the estimates derived using the factors' second indicators as referent indicators. Analogously, if necessary, we use $\widehat{\theta}^{\text {(FM3) }}$ to denote that the third indicator is the referent indicator and so on.

\subsection{Conversion Rules}

As a first application, we deal with the conversion of parameter estimates obtained under a given scaling method to the corresponding estimates obtained under another scaling method. The following proposition shows how to convert estimated parameters from one scaling method to another, without having to re-estimate the model. In this section, $\widehat{\theta}$ denotes the vector of estimated parameters under any scaling method and $\widehat{\hat{\theta}}$ denotes the vector of estimated parameters under another scaling method. ${ }^{13}$

Proposition 2 (Conversion Rules). If, for an up to scaling identified model, $\widehat{\hat{\theta}}$ and $\widehat{\theta}$ imply the same covariance matrix for the data, i.e., if $\boldsymbol{\Sigma}(\widehat{\hat{\theta}})=\boldsymbol{\Sigma}(\widehat{\theta})$, then we have: $\widehat{\theta} \stackrel{\boldsymbol{D}_{\xi}, \boldsymbol{D}_{\eta}}{\widehat{\hat{\theta}}}$, where $\boldsymbol{D}_{\xi}, \boldsymbol{D}_{\eta}$ are the diagonal matrices with diagonal elements $(i=1, \ldots, n ; j=1, \ldots, m)$

1. for $\widehat{\theta}=\widehat{\theta}^{(\mathrm{FM})}: d_{\xi, i i}=\frac{1}{\widehat{\hat{\lambda}}_{x, R_{\xi, i}, i}}$ and $d_{\eta, j j}=\frac{1}{\widehat{\hat{\lambda}}_{y, R_{\eta, j}, j}}$,

2. for $\widehat{\theta}=\widehat{\theta}^{(\mathrm{EC})}: d_{\xi, i i}=\frac{1}{\mathrm{~A}\left(\hat{\widehat{\lambda}}_{x, \bullet i}\right)}$ and $d_{\eta, j j}=\frac{1}{\mathrm{~A}\left(\hat{\widehat{\lambda}}_{y, \bullet j}\right)}$,

3. for $\widehat{\theta}=\widehat{\theta}^{(\mathrm{FF})}: d_{\xi, i i}=\sqrt{\widehat{\widehat{\phi}}_{i i}}$ and $d_{\eta, j j}=\sqrt{\widehat{\widehat{\psi}}_{j j}}$.

\footnotetext{
${ }^{13}$ Recall that reference indicators, $R_{\xi, i}$ and $R_{\eta, j}$, have been defined in Definition 3, while average factor loadings, $\mathrm{A}\left(\widehat{\hat{\lambda}}_{x, \bullet i}\right)$ and $\mathrm{A}\left(\widehat{\hat{\lambda}}_{y, \bullet j}\right)$, have been defined in Definition 4 .
} 
Proof. First of all, regardless of the scaling method employed, due to the model being identified up to scaling, there exist diagonal matrices $\boldsymbol{D}_{\xi}$ and $\boldsymbol{D}_{\eta}$ such that $\widehat{\theta} \stackrel{\boldsymbol{D}_{\xi}, \boldsymbol{D}_{\eta}}{\sim} \widehat{\hat{\theta}}$. The specific structure of those diagonal matrices follows from the restrictions which the scaling methods impose on $\widehat{\theta}(i=1, \ldots, n ; j=1, \ldots, m):^{14}$

1. for fixed marker scaling, $\widehat{\boldsymbol{\Lambda}}_{x}=\widehat{\hat{\boldsymbol{\Lambda}}}_{x} \boldsymbol{D}_{\xi}$ and $\widehat{\boldsymbol{\Lambda}}_{y}=\widehat{\hat{\boldsymbol{\Lambda}}}_{y} \boldsymbol{D}_{\eta}$ imply $1=\widehat{\lambda}_{x, R_{\xi, i}, i}=\widehat{\hat{\lambda}}_{x, R_{\xi, i}, i} d_{\xi, i i}$ and $1=\widehat{\lambda}_{y, R_{\eta, j}, j}=\widehat{\hat{\lambda}}_{y, R_{\eta, j}, j} d_{\eta, j j}$, from which the formulas given above are straightforwardly derived,

2. for effects coding scaling, $\widehat{\boldsymbol{\Lambda}}_{x}=\widehat{\widehat{\Lambda}}_{x} \boldsymbol{D}_{\xi}$ and $\widehat{\boldsymbol{\Lambda}}_{y}=\widehat{\widehat{\Lambda}}_{y} \boldsymbol{D}_{\eta}$ imply $1=\mathrm{A}\left(\widehat{\lambda}_{x, \bullet}\right)=$ $\mathrm{A}\left(\widehat{\hat{\lambda}}_{x, \bullet i}\right) d_{\xi, i i}$ and $1=\mathrm{A}\left(\widehat{\lambda}_{y, \bullet j}\right)=\mathrm{A}\left(\widehat{\hat{\lambda}}_{y, \bullet j}\right) d_{\eta, j j}$, from which the formulas given above are straightforwardly derived,

3. for fixed factor scaling, $\widehat{\boldsymbol{\Phi}}=\boldsymbol{D}_{\xi}^{-1} \widehat{\widehat{\boldsymbol{\Phi}}} \boldsymbol{D}_{\xi}^{-1}$ and $\widehat{\boldsymbol{\Psi}}=\boldsymbol{D}_{\eta}^{-1} \widehat{\hat{\mathbf{\Psi}}} \boldsymbol{D}_{\eta}^{-1}$ imply $1=\widehat{\phi}_{i i}=\frac{\widehat{\hat{\phi}}_{i i}}{d_{\xi, i i}^{2}}$ and $1=\widehat{\psi}_{j j}=\frac{\widehat{\widehat{\psi}}_{j j}}{d_{\eta, j j}^{2}}$, from which the formulas given above are straightforwardly derived.

Remark 5 (Conversion rules independent of scaling). A surprising fact revealed by Proposition 2 is that the conversion rules do not depend on the starting point for conversion, but only on the scaling method into which one wants to convert. The reason behind this property is that parameters estimated under two different scaling methods are related via a change of scale according to diagonal matrices with entries $d_{\xi, i i}$ and $d_{\eta, j j}$, whose values only depend on the values of $\widehat{\hat{\theta}}$, but not on the scaling method used to determine $\widehat{\hat{\theta}}$.

Remark 6 (Conversion to EV scaling). Given the assumptions of Proposition 2, it is also possible to convert a parameter $\widehat{\hat{\theta}}$ estimated under any scaling to the according parameter $\widehat{\theta}$ estimated under the EV scaling method mentioned in Remark 2. This is done by setting $d_{\xi, i i}=\frac{1}{\sum_{k=1}^{q} \widehat{\lambda}_{x, k i}^{2}}$ for an exogenous latent variable $\xi_{i}$ and $d_{\eta, j j}=\frac{1}{\sum_{l=1}^{p} \widehat{\lambda}_{y, l j}^{2}}$ for a latent endogenous variable $\eta_{j}$.

For the reader's convenience the formulas given in Proposition 2 are also summarized in Table 2. ${ }^{15}$ Table 2 should be read as follows: In the left-most column, the rows contain

\footnotetext{
${ }^{14}$ The proof of Proposition 2 uses some basic rules of matrix algebra. Firstly, the inverse of a diagonal matrix is again a diagonal matrix obtained by inverting the entries on the diagonal. Secondly, left multiplying a matrix by a diagonal matrix corresponds to a multiplication of the rows of the matrix by the respective entries in the diagonal matrix, while right multiplying a matrix by a diagonal matrix corresponds to a multiplication of the columns of the matrix by the respective entries in the diagonal matrix.

${ }^{15}$ In the supplementary material, we provide a corollary that states the conversion rules for parameters without using matrices and, moreover, an additional corollary referring to the conversion of indirect effects.
} 
the parameters we wish to determine. These parameters $\widehat{\theta}^{(\mathrm{S})}$ are designated with a hat and the superscript S is from the set FM, EC, and FF. The superscript stands for the "target" scaling methods, i.e., the estimates we wish to calculate given the estimates obtained under any scaling method to which we refer as $\widehat{\hat{\theta}}$. Thus, the formulas for conversion are provided in the columns with the label of the desired scaling method. The structure of the table reflects the property of the conversion rules that it only matters into which scaling method the estimated parameters are to be converted: thus, the column headers indicate the target scaling method that parameters are to be converted to, while the row headers determine which specific parameter is to be determined. For example, if we want to determine the estimated loading of indicator $k$ on the exogenous variable $i$ under the EC scaling method, i.e., $\hat{\lambda}_{x, k i}^{(\mathrm{EC})}$, the conversion formula is given in the first row and second column of Table 2. In section 3 , we will demonstrate in detail how to apply these conversion formulas. We conclude this subsection with a remark concerning the application of the conversion rules in misspecified models (correctly specified and misspecified models are introduced in the next section).

Remark 7 (Conversion rules in misspecified models). The conversion rules do not depend on the model being correctly specified. They hold for correctly specified models as well as for misspecified models. We will further elaborate on this property of the conversion formulas in section 3.3. Additionally, we also to point out that the choice of a particular scaling method has no influence at all on whether a model is correctly specified or not.

\subsection{Relations between Estimated and Population Quantities}

We now turn our attention to relations between estimated parameters and population values. As stated above, the scaling of a structural equation model is concerned with restrictions on the estimated parameters, but not with the underlying "true" or "population" parameters. In order to develop the correct interpretations of estimated parameters, however, it is essential to thoroughly differentiate between estimated and population quantities. In the population, there is a data generating process ( $\mathrm{dgp}$ ) and the measurement and structural equations assume that this dgp is described by linear functions. According to Pearl (2009, p. 27), these linear functions denote "a recipe, a strategy, or a law specifying what value nature would assign to" the dependent variables depending on the values of the independent variables and the residual errors. With regard to the interpretation, we assume that we have knowledge of the data generating process (cf. Bartholomew, Knott, \& Moustaki, 2011, p. 215), i.e., we assume that we have knowledge of the structure of the linear functions describing the dgp with their parameters $\theta^{\text {(pop) }}$, but do not know the population quantities of the parameters. This assumption is in line with the basic notion of structural equation models as a tool for testing 
theories. Wright (1923, p. 240) notes that "prior knowledge of the causal relations is assumed as a prerequisite." In the same vein, Grace (2006, p. 9) states that "structural equation modeling does not start with a null hypothesis that is given priority. Rather, structural equation models are built upon the complete body of available knowledge." These statements reveal that SEMs represent hypothetical relations between some entities that are measured with some latent variables and that these structures are the subject of investigation. However, there is nothing that provides any prior knowledge about the scales of the latent variables. This relation between dgps, SEM's, scaling, and estimated models is shown in Figure 4. In this figure, the upper left side depicts the real world, which a researcher does not know, and which is statistically represented by the population and the dgp working therein. The dgp is represented by the population parameter vector $\theta^{(\mathrm{pop})}$ and yields data that is observable and is drawn by means of a random sample. The researcher has a theory about the data generating process which he formalizes in terms of a SEM that is represented by the parameter vector $\theta$. The researcher's SEM that is encoded in $\theta$ is an unscaled model. Because there is no clue about the real scale of the latent variables in the unscaled model, the application of a scaling method is necessary. After applying a scaling method, the model can be estimated using the data that is given by the observations. A major point in Figure 4 is the relation between the dgp and the researcher's model: we assume that the researcher's model matches the dgp, i.e., we assume that the researcher has specified his model in accordance with the dgp. Thus, drawing on a realist perspective, the theory of the researcher has a correspondence with the real world (Pratschke, 2003). We arrive at the following assumption:

Assumption 3 (Correctly Specified). The model specified by the researcher matches the dgp in the population. In this case, we say that the model is correctly specified.

Assumption 3 can be restated to say that the graphical model that depicts the dgp is the same as the graphical model that depicts the model that is specified by the researcher. Under this assumption, we can specify the data generating process as a SEM by applying standard restrictions on the model matrices. That is, non-existing relations between model parameters are encoded by vanishing entries in the corresponding matrices. This results in the model matrices in the researcher's model having the same structure as those in the dgp. Should the dgp and the specified model not have the same structure, the last assumption refers to what is known as specification error (e.g., Kaplan, 2000).

Due to chance as well as scaling, estimated parameters $\widehat{\theta}$ and population parameters $\theta^{\text {(pop) }}$ will typically take different values. However, there will be a relation between population parameters and their estimated versions when the sample covariance matrix is close to the population covariance matrix, which is explored in the following proposition. 
Proposition 3. If, under Assumption 3, the sample covariance matrix $\boldsymbol{S}$ is close to the population covariance matrix $\boldsymbol{\Sigma}\left(\theta^{(\mathrm{pop})}\right)$ for an up to scaling identified structural equation model, then $\widehat{\theta}$ and $\theta^{(\mathrm{pop})}$ are approximately linked via a change of scale and

$$
\begin{aligned}
\widehat{\boldsymbol{\Lambda}}_{x} \approx \boldsymbol{\Lambda}_{x}^{(\mathrm{pop})} \boldsymbol{D}_{\xi}, \quad \widehat{\boldsymbol{\Lambda}}_{y} \approx \boldsymbol{\Lambda}_{y}^{(\mathrm{pop})} \boldsymbol{D}_{\eta}, \quad \widehat{\boldsymbol{\Gamma}} \approx \boldsymbol{D}_{\eta}^{-1} \boldsymbol{\Gamma}^{(\mathrm{pop})} \boldsymbol{D}_{\xi}, \quad \widehat{\boldsymbol{B}} \approx \boldsymbol{D}_{\eta}^{-1} \boldsymbol{B}^{(\mathrm{pop})} \boldsymbol{D}_{\eta} \\
\widehat{\boldsymbol{\Phi}} \approx \boldsymbol{D}_{\xi}^{-1} \boldsymbol{\Phi}^{(\mathrm{pop})} \boldsymbol{D}_{\xi}^{-1}, \quad \widehat{\boldsymbol{\Psi}} \approx \boldsymbol{D}_{\eta}^{-1} \boldsymbol{\Psi}^{(\mathrm{pop})} \boldsymbol{D}_{\eta}^{-1}, \quad \widehat{\boldsymbol{\Theta}}_{\delta} \approx \boldsymbol{\Theta}_{\delta}^{(\mathrm{pop})}, \quad \widehat{\boldsymbol{\Theta}}_{\epsilon} \approx \boldsymbol{\Theta}_{\epsilon}^{(\mathrm{pop})}
\end{aligned}
$$

where $\boldsymbol{D}_{\xi}, \boldsymbol{D}_{\eta}$ are the diagonal matrices with diagonal elements $(i=1, \ldots, n ; j=1, \ldots, m)$

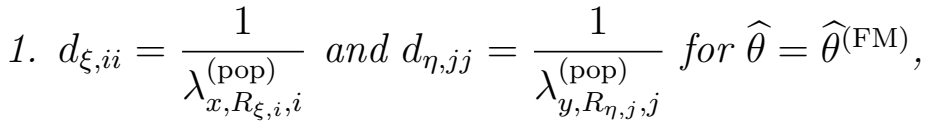

$$
\begin{aligned}
& \text { 2. } d_{\xi, i i}=\frac{1}{\mathrm{~A}\left(\lambda_{x, \bullet i}^{(\mathrm{pop})}\right)} \text { and } d_{\eta, j j}=\frac{1}{\mathrm{~A}\left(\lambda_{y, \bullet j}^{(\mathrm{pop})}\right)} \text { for } \widehat{\theta}=\widehat{\theta}^{(\mathrm{EC})} \text {, } \\
& \text { 3. } d_{\xi, i i}=\sqrt{\phi_{i i}^{(\mathrm{pop})}} \text { and } d_{\eta, j j}=\sqrt{\psi_{j j}^{(\mathrm{pop})}} \text { for } \widehat{\theta}=\widehat{\theta}^{(\mathrm{FF})} \text {. }
\end{aligned}
$$

Proof. Due to $\boldsymbol{S}$ being close to $\boldsymbol{\Sigma}\left(\theta^{(\mathrm{pop})}\right), \theta^{(\mathrm{pop})}$ and $\widehat{\theta}$ are approximately related via a change of scale. The proof then proceeds along exactly the same lines as the proof of Proposition 2.

Remark 8 (Manifest residual errors). The preceding proposition gives the relation between population and estimated parameters. Especially, it states that under Assumption 3, all elements of the manifest residual error covariances $\widehat{\boldsymbol{\Theta}}_{\delta}$ and $\widehat{\boldsymbol{\Theta}}_{\epsilon}$, whenever they are not fixed a priori, are always estimated as the population quantity. With regard to fixed elements of $\widehat{\boldsymbol{\Theta}}_{\delta}$ and/or $\widehat{\boldsymbol{\Theta}}_{\epsilon}$, this assertion only holds if these elements are fixed to their population value. If they were fixed to any other value, this would be a misspecification, yielding the estimates to deviate from the population quantity. This is also reflected in Proposition 3, which explicitly states that Assumption 3 must hold, thus precluding misspecified models.

Remark 9 (Estimated parameters under the EV scaling). Given the assumptions of Proposition 3, the relation of the estimated parameters under the EV scaling (see Remark 2) and their population quantities follows when choosing $d_{\xi, i i}=\frac{1}{\sum_{k=1}^{q}\left(\lambda_{x, k i}^{(\mathrm{pop})}\right)^{2}}$ and $d_{\eta, j j}=\frac{1}{\sum_{l=1}^{p}\left(\lambda_{x, l j}^{(\mathrm{pop})}\right)^{2}}$.

Remark 10 (Limit estimates). When the sample size increases to infinity for a correctly specified model, then $\boldsymbol{S}$ converges to $\boldsymbol{\Sigma}\left(\theta^{(\mathrm{pop})}\right)$ and the approximate relations in Proposition 3 come extremely close to equalities. Therefore, the preceding results show to which transformations of the population parameters estimators will converge, depending on the scaling method employed. These quantities will hereafter be called limit estimates. 
For the sake of convenience, we summarized the formulas for calculating the limit estimates in Table $3{ }^{16}$ In this table, the left-most column shows the estimated parameter under consideration and the other columns contain the formulas for each parameters estimated under the scaling method indicated in column's header. For instance, Table 3 shows that under FM scaling, $\widehat{\lambda}_{x, k i}$ is an estimate of the ratio of the population loading $\lambda_{x, k i}^{(\mathrm{pop})}$ of the $k$-th indicator over the population loading $\lambda_{x, R_{\xi, i}, i}^{(\mathrm{pop})}$ of the referent indicator of the $i$-th latent variable $\xi_{i}$.

\subsection{Summarizing the Math}

In this section, we provide a short non-technical summary of the major topics covered in this section.

In subsection 2.1, we firstly introduced the full LISREL model as a generic notation to represent SEMs. The term "generic" means that this notation refers to all types of models, where the parameter vector $\theta$ may either contain the population parameters, the parameters in a model specified by a researcher, or even the parameters estimated under some scaling method. In Definition 1, we then introduced the concept of a change of scale which states that the two generic parameter vectors $\theta$ and $\widetilde{\theta}$ are related via a change of scale if and only if they correspond to situations where the latent, unobservable variables in one model are simply rescaled versions of the corresponding latent, unobservable variables in the other model.

Secondly, we then developed the key property of changes of scale: whenever two parameter vectors are related via a change of scale, they necessarily imply the same covariance matrix of the manifest indicators.

Thirdly, we introduced the notion of unscaled and scaled SEMs. This distinction separates original models without scaling conditions being imposed from the corresponding, deduced scaled models which emanate from the unscaled models by adding scaling restrictions. The necessity to scale unscaled models arises from the fact that for unscaled models, parameter estimates can not be uniquely determined. Correspondingly, for unscaled models, we introduced in Definition 2 the concept of "identified up to scaling": it simply states that an unscaled model is identified up to scaling if and only if adding scaling restrictions to it renders the model identified. Obviously, this is tantamount to stating that all non-uniqueness and identification problems of an up to scaling identified unscaled model stem from the fact that it lacks scaling. Thus, applying a scaling method to an up to scaling identified (unscaled) model renders that model identified.

\footnotetext{
${ }^{16}$ In the supplementary material, we provide a corollary that provides a less compact, more reader-friendly form of Proposition 3. The supplement also contains a proposition regarding indirect effects and a proposition regarding the invariance of $R^{2}$ with respect to the scaling method.
} 
Finally, we provided formal definitions of the three common scaling methods. All these definitions draw on fixing estimated parameters (FM, FF) or enforcing restrictions involving several estimated parameters (EC). With respect to notation, we used "hats", e.g. $\widehat{\theta}$, to make it clear when we are concerned with estimated parameters, and we used superscripts to distinguish between scaling methods, resulting, e.g., in the notations $\widehat{\theta}^{(\mathrm{FM})}, \widehat{\theta}^{(\mathrm{EC})}$, and $\widehat{\theta}^{(\mathrm{FF})}$.

In subsection 2.2, we developed conversion rules. These rules show how to convert parameters estimated under one scaling method into parameters estimated under a different scaling method, without the need to reestimate the model. The conversion rules make use of the fact that the corresponding parameter vectors are related by a change of scale, see the explanation in Remark 5. Furthermore, it turned out that the conversion rules only depend on the target scaling method, but not on the initially used scaling method. Additionally, these rules are applicable even when the model is not correctly specified.

With regard to the relation between estimated parameters and population parameters, we introduced the notation with the superscript "(pop)" in subsection 2.3 to clearly distinguish the population parameters $\theta^{(\mathrm{pop})}$ from other generic parameters $\theta$ as well as from estimated

parameters $\hat{\theta}$. In order to ensure that estimated and population parameters are actually related to each other, a correctly specified model is essential, as stated in Assumption 3. Under this additional prerequisite, population and estimated parameter vectors are related by a change of scale, entailing that specific estimated parameters such as loadings and regression coefficients constitute algebraic transformations of certain population parameters which are summarized in Table 3.

The fact that estimated parameters are algebraic transformations of population quantities causes issues concerning the estimated parameters' interpretation and their statistical tests. These issues will be outlined in the next two sections.

\section{Exemplifying Theoretical Results and Interpreting Parameter Estimates}

In this section, we will explain the results from the previous section using the regression example from Figure 2. In subsection 3.1 we demonstrate how to convert the estimated parameters obtained under one scaling method to their equivalent obtained under another scaling method. In subsection 3.2, we exemplify the interpretation of the estimated parameters for all three scaling methods, extending the interpretation of estimated parameters described 
in Klößner and Klopp (2019). ${ }^{17}$ Finally, in subsection 3.3, we deal with the issue of the interpretation of estimated parameters in the context of misspecified models.

\subsection{Conversion of Estimated Parameters}

In order to illustrate the conversion of estimated parameters, we consider the regression model from the introduction depicted in Figure 2 and the summary of the conversion formulas from Proposition 2 in Table 2. In this section we will use the limit estimates of the estimated parameters according to Remark 10. We use the limit estimates in order to minimize potential rounding errors and to ease the readers understanding of the applications of our formulas. However, in real world applications, the conversion as well as the interpretation of the parameters would certainly rest on the estimates. The limit estimates for the regression example are shown in Table 5. These are obtained by using the formulas from Table 3 and putting in the population quantities of the model parameters from Figure 2. Moreover, we look at the case of FM1 scaling and the conversion to EC scaling. Thus, the parameters under EC scaling take the role of the parameters with the hat, $\widehat{\theta}$, in the leftmost column in Table 2 and the estimated parameters under FM1 scaling are parameters with the double hat, $\widehat{\hat{\theta}}$, in column label "Effects coding". Note that it is possible to convert from each scaling method to each other. However, we do not demonstrate this, but provide an overview of almost all possible conversions in Table 6 . In this table, we leave aside FM2 scaling because the conversion from and to FM2 scaling is analogous to the FM1 case.

We start with the conversion of the factor loading of the exogenous latent variable $\xi_{1}$. In the first step, we calculate the average of the estimated factor loadings obtained under FM1 of $\xi_{1}$, which is $\mathrm{A}\left(\widehat{\hat{\lambda}}_{x, 11}, \widehat{\hat{\lambda}}_{x, 21}\right)=\frac{1.000+2.000}{2.000}=1.500$. In the second step, to obtain the estimated loadings under the EC scaling, we divide the estimated loadings under FM1 scaling by this average. For the first indicator, we obtain $\widehat{\lambda}_{x, 11}^{(\mathrm{EC})}=\frac{1.000}{1.500}=0.667$, and for the second indicator $\widehat{\lambda}_{x, 21}=\frac{2.000}{1.500}=1.333$, which are the exact values found in Table 5 . The same conversion procedure applies to the loadings of the endogenous latent variable. To convert the estimated regression coefficient, we have to multiply the ratio of the average factor loadings under FM1 scaling by the estimated regression coefficient under FM1 scaling, which yields $\widehat{\gamma}_{11}=\frac{0.5625}{1.500} \cdot 12.000=4.500$. The variance of the exogenous latent variable under the EC scaling method is given by $\widehat{\phi}_{11}=1.500^{2} \cdot 1.000=2.250$, and the variance of the endogenous residual is $\widehat{\psi}_{11}=0.5625^{2} \cdot 64.000=20.250$.

\footnotetext{
${ }^{17}$ The $\mathrm{R}$ code for all examples is available on OSF (https://osf.io/4rwkt/).
} 


\subsection{Interpretation of Estimated Parameters}

Next, we demonstrate how to interpret estimated parameters. For this demonstration, we use the results from Proposition 3 and the summary of its formulas given in Table 3, building on the regression model and the limit estimates given in Figure 2 and Table 5, respectively. In the following, we explain the interpretation of the estimated parameters under the FM, EC, and FF scaling method. In order to summarize the interpretation, we provide short and general rules how to interpret each parameter under the three scaling methods in Table 4 . At the end of this subsection, we discuss the consequences of the relation between population and estimated parameters.

Starting with the interpretation of the estimates obtained under the FM1 scaling method, again, we only consider the case when the first indicator is used as the referent indicator. The estimated factor loading of an indicator is given by the ratio of the population loading of this indicator over the population loading of the referent indicator, which is $\widehat{\lambda}_{x, 21}^{(\mathrm{FM} 1)}=\frac{2.000}{1.000}=2.000$ for the second loading of the exogenous latent variable. The interpretation is as follows: Indicator $x_{21}$ loads 2 times stronger on the latent variable $\xi_{1}$ than the referent indicator $x_{11}$ does. The same principle applies to the estimated loading of the second indicator $y_{21}$ on the latent endogenous variable $\eta_{1}$ : the estimated loading is $\widehat{\lambda}_{y, 21}^{(\mathrm{FM} 1)}=\frac{0.500}{4.000}=0.125$, i.e., the indicator $y_{21}$ loads 0.125 times as strong on the latent variable $\eta_{1}$ as the referent indicator does. The general rule for interpreting loadings estimated under FM1 scaling is, thus, that they indicate how strongly the corresponding indicator loads on the latent variable relative to the latent variable's reference indicator.

The interpretation of estimated regression coefficients is not as straightforward as the interpretation of factor loadings. The estimated regression coefficient indicates that a one unit change $\Delta \xi_{1}$ of the exogenous latent variable $\xi_{1}$ yields a $\widehat{\gamma}_{11}^{(\mathrm{FM} 1)}=12.000$ change of the endogenous latent variable $\eta_{1}$ in the scale set by the FM1 scaling. Therefore, we first have to investigate what this means: Building on Equation (1) and a ceteris paribus assumption that all other quantities stay unchanged, we have the following relation between $\Delta x_{11}$, the size of change of $x_{11}$, and $\Delta \xi_{1}$, the size of change of $\xi_{1}$ :

$$
\Delta x_{11}=\lambda_{x, 11}^{(\mathrm{pop})} \cdot \Delta \xi_{1}
$$

This implies that $\Delta x_{11}=1$ if and only if $\Delta \xi_{1}=\frac{1}{\lambda_{x, 11}^{(\text {pop })}}$. Thus, a one unit change of $\xi_{1}$ in the scale set by the FM1 scaling is tantamount to $\xi_{1}$ changing by $\Delta \xi_{1}=\frac{1}{\lambda_{x, 11}^{(\text {pop })}}$ in its own, 
unknown scale. The associated change $\Delta \eta_{1}$ of the exogenous latent variable $\eta_{1}$ is then

$$
\Delta \eta_{1}=\gamma_{11}^{(\mathrm{pop})} \cdot \Delta \xi_{1}=\gamma_{11}^{(\mathrm{pop})} \cdot \frac{1}{\lambda_{x, 11}^{(\mathrm{pop})}}
$$

and accordingly, the referent indicator of the endogenous latent variable $\eta_{1}$ changes by

$$
\Delta y_{11}=\lambda_{y, 11}^{(\mathrm{pop})} \cdot \Delta \eta_{1}=\frac{\lambda_{y, 11}^{(\mathrm{pop})}}{\lambda_{x, 11}^{(\mathrm{pop})}} \cdot \gamma_{11}^{(\mathrm{pop})}
$$

Consequently, the interpretation of $\widehat{\gamma}_{11}^{(\mathrm{FM} 1)}$, whose limit estimate is given by $\frac{\lambda_{y, 11}^{(\mathrm{pop})}}{\lambda_{x, 11}^{(\mathrm{pop})}} \cdot \gamma_{11}^{\text {(pop) }}$, is as follows: When the exogenous latent variable $\xi_{1}$ changes in such a way that its referent indicator $x_{11}$ changes by one unit, then the endogenous latent variable $\eta_{1}$ changes in such a way that its referent indicator $y_{11}$ changes by $\widehat{\gamma}_{11}^{(\mathrm{FM} 1)}$ units. As $\widehat{\gamma}_{11}^{(\mathrm{FM} 1)}$ equals 12.000 for the example at hand, we can state that when $\xi_{1}$ changes such that its referent indicator $x_{11}$ changes by one unit, then the endogenous latent variable $\eta_{1}$ will change in such a way that its referent indicator $y_{11}$ changes by 12.000 units. Although this example only contains the regression of an endogenous latent variable on an exogenous latent variable, the interpretation straightforwardly also applies to the estimated regression of an endogenous latent variable on another endogenous latent variable.

The interpretation of the estimated variances and covariances of the latent variables differs from the interpretation of the estimated loadings and regression coefficients. Due to $x_{11}=$ $\lambda_{x, 11}^{(\mathrm{pop})} \xi_{1}+\delta_{11}$, the interpretation of the estimated variance $\widehat{\phi}_{11}^{(\mathrm{FM} 1)}$, whose limit estimate is $\left(\lambda_{x, 11}^{(\text {pop })}\right)^{2} \cdot \phi_{11}^{(\mathrm{pop})}$, is that $\widehat{\phi}_{11}^{(\mathrm{FM} 1)}=1.000$ describes the amount of variance of the indicator $x_{11}$ which can be attributed to the latent variable $\xi_{1}$. The general rule is that an exogenous latent variable's estimated variance under FM scaling can be interpreted as the amount of variance that this latent variable transports to its referent indicator. By similar considerations, the interpretation of estimated covariances between exogenous latent variables is that they estimate the amount of covariance which these latent variables transmit to their referent indicators.

Attention has to be paid to the fact that this interpretation does not apply to the endogenous latent variables, as $\widehat{\psi}_{11}^{(\mathrm{FM} 1)}$ does not refer to the estimated variance of the endogenous variable $\eta_{1}$, but to the estimated variance of its disturbance $\zeta_{1}$. However, it is easy to see that

$$
y_{11}=\lambda_{y, 11}^{(\mathrm{pop})} \eta_{1}+\varepsilon_{11}=\lambda_{y, 11}^{(\mathrm{pop})}\left(\gamma_{11}^{(\mathrm{pop})} \xi_{1}+\zeta_{1}\right)+\varepsilon_{11}=\lambda_{y, 11}^{(\mathrm{pop})} \gamma_{11}^{(\mathrm{pop})} \xi_{1}+\lambda_{y, 11}^{(\mathrm{pop})} \zeta_{1}+\varepsilon_{11},
$$


from which we find that $\left(\lambda_{y, 11}^{(\mathrm{pop})}\right)^{2} \cdot \psi_{11}^{(\mathrm{pop})}$, the limit estimate of $\widehat{\psi}_{11}^{(\mathrm{FM} 1)}$, denotes the amount of variance of $y_{11}$ emanating from $\zeta_{1} \cdot{ }^{18}$ Therefore, the interpretation of $\widehat{\psi}_{11}^{(\mathrm{FM} 1)}=64.000$ is that it describes the amount of variance of $y_{11}$ caused by $\zeta_{1}$. The general rule for interpreting latent endogenous variables' variances estimated under the fixed marker method is that they estimate the amount of variance of the latent variable's referent indicator which is caused by the residual term appearing in the latent endogenous variable's equation. Similarly, estimating latent covariances using the fixed marker method leads to values which are to be interpreted as the amount of covariance that the two latent variables' disturbances transmit to the latent variables' referent indicators.

As Table 3 reveals, the limit estimates for the effects coding scaling method are structurally identical to those of the fixed marker method, with the reference indicators' loadings $\lambda_{x, R_{\xi, i}, i}^{\text {(pop }}$ and $\lambda_{y, R_{\eta, j}, j}^{(\text {pop })}$ being replaced by $\mathrm{A}\left(\lambda_{x, \bullet i}^{(\mathrm{pop})}\right)$ and $\mathrm{A}\left(\lambda_{y, \bullet j}^{(\mathrm{pop})}\right)$, respectively. For this reason, in order to develop interpretations for the estimates obtained under EC scaling, we only need to find an interpretation for $\mathrm{A}\left(\lambda_{x, \bullet i}^{(\text {pop })}\right)$ and $\mathrm{A}\left(\lambda_{y, \bullet j}^{(\text {pop })}\right)$, which can then replace the referent indicator loadings in the interpretations for estimates calculated under FM scaling. In order to derive this interpretation of $\mathrm{A}\left(\lambda_{x, \bullet i}^{(\mathrm{pop})}\right)$ and $\mathrm{A}\left(\lambda_{y, \bullet j}^{(\mathrm{pop})}\right)$ for the example at hand, we define new pseudoindicators $\overline{x_{1}}:=\frac{1}{2}\left(x_{11}+x_{21}\right)$ and $\overline{y_{1}}:=\frac{1}{2}\left(y_{11}+y_{21}\right)$, which we call the 'average indicators' of the indicators of the latent variables $\xi_{1}$ and $\eta_{1} \cdot{ }^{19}$ From $x_{11}=\lambda_{x, 11}^{(\mathrm{pop})} \xi_{1}+\delta_{11}$ and $x_{21}=$ $\lambda_{x, 21}^{(\mathrm{pop})} \xi_{1}+\delta_{21}$, we find that

$$
\begin{aligned}
\overline{x_{1}} & =\frac{1}{2}\left(x_{11}+x_{21}\right)=\frac{1}{2}\left(\lambda_{x, 11}^{(\mathrm{pop})} \xi_{1}+\delta_{11}+\lambda_{x, 21}^{(\mathrm{pop})} \xi_{1}+\delta_{21}\right) \\
& =\frac{1}{2}\left(\lambda_{x, 11}^{(\mathrm{pop})}+\lambda_{x, 21}^{(\mathrm{pop})}\right) \xi_{1}+\frac{1}{2}\left(\delta_{11}+\delta_{21}\right)=\mathrm{A}\left(\lambda_{x, \bullet i}^{(\mathrm{pop})}\right) \xi_{1}+\frac{1}{2}\left(\delta_{11}+\delta_{21}\right),
\end{aligned}
$$

i.e., the average indicator loads $\overline{x_{1}}$ loads on the latent variable $\xi_{1}$ according to $\mathrm{A}\left(\lambda_{x, \bullet 1}^{(\mathrm{pop})}\right)$. Similar derivations show that $\overline{y_{1}}=\mathrm{A}\left(\lambda_{y, \bullet 1}^{(\mathrm{pop})}\right) \eta_{1}+\frac{1}{2}\left(\epsilon_{11}+\epsilon_{21}\right)$. Therefore, the interpretations for estimated parameters obtained using EC scaling are almost identical to those for FM scaling, the only difference being that all instances of 'reference indicator' are being replaced by 'average indicator', see Table 4 .

The interpretation of the estimated parameters obtained under the FF scaling method differs considerably from the interpretation of the estimated parameters under the FM or EC

\footnotetext{
${ }^{18}$ Please be aware that in Equation 10, the parameters referring to the error terms for both the manifest variables and the latent endogenous variable do not have a pop superscript. This is because these model are random variables and only the respective variances are part of the model. This can be seen in the model's parameter vector $\theta$ in Equation 5, as only the manifest residual variances and latent endogenous variances are elements of the parameter vector.

${ }^{19}$ In general, for latent variables on which more than two indicators may load, 'average indicators' are defined by calculating the arithmetic average of all its indicators.
} 
scaling method. This is because FM and EC scaling operate on the estimated factor loadings, whereas FF scaling operates on the estimated variances of the exogenous latent variables and the estimated variances of the residuals of the endogenous latent variables, which in order to achieve identification are set to 1 , respectively. In order to develop the interpretation of estimated loadings, we consider a ceteris paribus change of the exogenous latent variable $\xi_{1}$ by $\Delta \xi_{1}:=\sqrt{\phi_{11}^{(\mathrm{pop})}}$, which consequently induces indicator $x_{21}$ to change by

$$
\Delta x_{21}=\lambda_{x, 21}^{(\mathrm{pop})} \cdot \Delta \xi_{1}=\lambda_{x, 21}^{(\mathrm{pop})} \cdot \sqrt{\phi_{11}^{(\mathrm{pop})}}=\widehat{\lambda}_{x, 21}^{(\mathrm{FF})}
$$

Thus, the interpretation of $\widehat{\lambda}_{x, 21}^{(\mathrm{FF})}=2.000$ is as follows: when the latent variable $\xi_{1}$ changes by one standard deviation, then indicator $x_{21}$ changes by 2.000 units. The general rule thus is that loadings of exogenous latent obtained under FF scaling estimate by how much indicators change when the latent exogenous variable they are loading on changes by one standard deviation. ${ }^{20}$ Stated differently, when a latent exogenous variable changes by one standard deviation in its unknown scale, then its indicator changes approximately by $\widehat{\lambda}_{x, 21}^{(\mathrm{FF})}$ units. This is equivalent to saying that a one unit change of the latent variable in the scale set by the $\mathrm{FF}$ scaling method leads to its indicator changing by an estimated $\widehat{\lambda}_{x, 21}^{(\mathrm{FF})}$ units.

We will now turn our attention to the interpretation of loadings linked to endogenous latent variables which have been estimated under FF scaling. As the limit estimate of $\widehat{\lambda}_{y, 11}^{(\mathrm{FF})}$ is given by $\lambda_{y, 11}^{(\mathrm{pop})} \cdot \sqrt{\psi_{11}^{(\mathrm{pop})}}$, there is a structural similarity to the interpretation of exogenous latent variables' estimated loadings, the only difference being that $\sqrt{\psi_{11}^{(\mathrm{pop})}}$ does not refer to the latent variable's standard deviation, but to the standard deviation of its residual. Thus, the interpretation of $\widehat{\lambda}_{y, 11}^{(\mathrm{FF})}=8.000$ is as follows: when the latent endogenous variable $\eta_{1}$ changes by one standard deviation of its residual, then the indicator $y_{11}$ changes by 8.000 units. The general rule is, then, that loadings of endogenous latent obtained under FF scaling estimate by how much indicators change when the latent endogenous variable they are loading on changes by one standard deviation of its residual. ${ }^{21}$ In other words, when a latent endogenous variable changes in its unknown scale by one standard deviation of its residual, then its indicator changes approximately by the corresponding loading estimated

${ }^{20}$ Due to the identity $\lambda_{x, 21}^{(\mathrm{pop})} \cdot \sqrt{\phi_{11}^{(\mathrm{pop})}}=\sqrt{\left(\lambda_{x, 21}^{(\mathrm{pop})}\right)^{2} \cdot \phi_{11}^{(\mathrm{pop})}}$, there exists a second possibility of interpreting the estimated loading $\widehat{\lambda}_{x, 21}^{(\mathrm{FF})}$ : it estimates the square root of the amount of variance transmitted from the latent variable $\xi_{1}$ to its indicator $x_{21}$.

${ }^{21}$ Due to the identity $\lambda_{y, 11}^{(\mathrm{pop})} \cdot \sqrt{\psi_{11}^{(\mathrm{pop})}}=\sqrt{\left(\lambda_{y, 11}^{(\mathrm{pop})}\right)^{2} \cdot \psi_{11}^{(\mathrm{pop})}}$, there exists a second possibility of interpreting the estimated loading $\widehat{\lambda}_{y, 11}^{(\mathrm{FF})}$ : it estimates the square root of the amount of variance transmitted from the latent variable $\eta_{1}$ to its indicator $y_{11}$. 
under FF scaling. This is equivalent to saying that a one unit change of the latent variable in the scale set by the FF scaling method leads to its indicator changing by an estimated $\widehat{\lambda}_{y, 11}^{(\mathrm{FF})}$ units.

With respect to the interpretation of the estimated regression coefficient $\widehat{\gamma}_{11}^{(\mathrm{FF})}$, these considerations directly lead to the following conclusion: Under a ceteris paribus assumption that all other quantities stay unchanged, the estimated regression coefficient indicates by how many standard deviations of its residual the latent variable $\eta_{1}$ will change when the exogenous latent variable $\xi_{1}$ changes by one standard deviation. The interpretation of estimated regression coefficients $\widehat{\beta}_{j i}^{(\mathrm{FF})}$ is analogous, but not exactly the same. These estimated regression coefficients indicate by how many standard deviations of its residual $\zeta_{j}$ the endogenous variable $\eta_{j}$ will change when the endogenous variable $\eta_{i}$ changes by one standard deviation of its residual $\zeta_{j}$. Thus, the interpretation of the estimated regression coefficients bears some similarity to the interpretation of standardized regression coefficients.

As $\widehat{\phi}_{i i}^{(\mathrm{FF})}$ and $\widehat{\psi}_{j j}^{(\mathrm{FF})}$ are fixed to unity by FF scaling, there is no need for interpreting these quantities. Finally, as is evident, estimated covariances $\widehat{\phi}_{i_{1} i_{2}}^{(\mathrm{FF})}$ can be interpreted as correlations between the corresponding latent variables $\xi_{i_{1}}$ and $\xi_{i_{2}}$. Similarly, estimated covariances $\widehat{\psi}_{j_{1} j_{2}}^{(\mathrm{FF})}$ can be interpreted as correlations between the corresponding latent variables' residuals $\zeta_{j_{1}}$ and $\zeta_{j_{2}}$.

In Table 4, we have summarized the interpretations in a verbal form. The table therefore provides a collection of template-style wordings that can be applied in any specific research context. When using these templates, one simply inserts the actual estimate for the 'hatted' Greek letters denoting the parameters. For instance, the above interpretations of the estimated regression coefficients can be read from the table by inserting the numerical values for the estimated regression coefficients $\widehat{\gamma}$.

To conclude this subsection, we would like to draw the reader's attention to the fact that estimated regression coefficients never actually estimate the population regression coefficients. Also, there is no way to estimate the actual population regression coefficient because there is an infinite number of population quantities that yield the same estimated regression coefficient. The same holds true for most estimated parameters ${ }^{22}$ and is due to the latent variables' scales being unknown. When latent variables are scaled by using the FM or EC scaling, then the estimated regression coefficients involve some functions of the corresponding latent variables' loadings, while for the FF scaling, estimated coefficients depend on the latent variables' unknown variances. Thus, the actual interpretation always occurs in the metric set by the

\footnotetext{
${ }^{22}$ The only parameters not affected by changing the latent variables' scale are the variances and covariances of the manifest variables' residuals, i.e., the entries in $\boldsymbol{\Theta}_{\delta}$ and $\boldsymbol{\Theta}_{\epsilon}$. Thus, these parameters are the only ones that are not affected by the choice of scaling method.
} 
scaling method used (see the example above for the interpretation of the estimated regression coefficient under FM1 scaling: a one unit change of the exogenous latent variable $\xi_{1}$ yields a $\widehat{\gamma}_{11}^{(\mathrm{FM} 1)}$ units change of the endogenous latent variable $\eta_{1}$ in the scale set by the FM1 scaling). However, the interpretation in terms of the population quantities involved matters when a hypothesis about such a parameter is to be tested. For instance, when the hypothesis that the regression between the exo- and endogenous variables equals zero is tested, then the test of this hypothesis depends on the scaling (see Klößner \& Klopp, 2019). We will come back to and elaborate on this issue in the fourth section.

\subsection{Conversion, limit estimates, and misspecified models}

In this section, we consider a case when Assumption 3 is violated, i.e. when the graph of the dgp does not match the graph of the specified model. As an example, we consider a mediation model, the dgp of which is shown in Figure 5. The misspecified model used for estimation is shown in Figure 6. In the specified model, the indicators of $\eta_{2}$ are mistakenly assumed to load on $\eta_{1}$, and in turn only the regression between $\xi_{1}$ and $\eta_{1}$ is modeled. As can be seen in Figure 5, the measurement part of the exogenous variable corresponds to the measurement model in the dgp in Figure 6, as the graphs of those parts match. However, the measurement part of the endogenous variables does not match, because there are two latent variables in the population model, but only one in the misspecified model. As a consequence hereof the structural model does not match, either.

As stated in Remark 7, converting estimated parameters from one scaling method to another is perfectly possible, both for the correctly specified and misspecified parts of the model. For instance, to convert the estimated loading $\hat{\lambda}_{x, 2}^{(\mathrm{FM} 1)}$ to $\widehat{\lambda}_{x, 2}^{(\mathrm{FF})}$, we have according to Proposition 2, $\widehat{\lambda}_{x, 2}^{(\mathrm{FF})}=\widehat{\lambda}_{x, 2}^{(\mathrm{FM} 1)} \cdot \sqrt{\widehat{\phi}_{11}^{(\mathrm{FM} 1)}}=0.500 \cdot \sqrt{4.000}=1.000$. The same holds for the conversion $\widehat{\gamma}_{11}^{(\mathrm{FF})}=\frac{\sqrt{\widehat{\phi}_{11}^{(\mathrm{FM} 1)}}}{\sqrt{\widehat{\psi}_{11}^{(\mathrm{FM} 1)}}} \cdot \widehat{\gamma}_{11}^{(\mathrm{FF})}=\frac{\sqrt{4.000}}{\sqrt{24.977}} \cdot 1.841=0.737$.

The possibility to convert the estimates stemming from the misspecified part illustrates that to apply the formulas from Proposition 2, it is only necessary that the models under the various scaling methods have the same model-implied covariance matrix. Then they are related via change of scale and the conversion formulas are applicable. In contrast, Proposition 3 rests additionally on Assumption 3. If this assumption is violated, then the relations stated in Proposition 3 vanishes.

Concerning the estimated parameters, we have to differentiate the parts of the model in 6 that match, or respectively, do not match the dgp. We will now demonstrate that the estimates adhere to the formulas for the limit estimates from Proposition 3 for the matching 
part (see also Table 3), whereas the relation between the estimated parameters and the limit estimates vanishes for the part that does not match.

For this demonstration, we use the population covariance matrix (see Appendix B) to estimate the model with a sample size of $N=500$. For the sake of simplicity, we consider only the results under FM1 scaling. It could be expected for a correctly specified model that the estimates are identical to the limit estimates (apart from potential minimal deviations due to approximation and rounding). As the fit measures reveal, the model does not have a particular good fit to the data, $\chi^{2}(26)=67.946, p \leq .001$, CFI $=0.980, \mathrm{SRMR}=0.025$, RMSEA $=0.057$, which indicates that there are serious problems with this model, thus providing evidence that the model should be discarded. However, investigating the estimated parameters shown in Table 7 reveals that for the part matching the dgp the limit estimates behave according to Proposition 3. For instance, in line with the formulas for the estimated loading, we have $\hat{\lambda}_{x, 2}^{(\mathrm{FM} 1)}=\frac{1}{2}=0.500$, which exactly equals the actually estimated parameter value. The same holds true for the estimated variance $\widehat{\phi}_{11}^{(\mathrm{FM} 1)}$, which is very close to its limit estimate.

Additionally, all estimated manifest residual error variances, in line with Remark 1, do not depend on the scaling method. Furthermore, the residual variances belonging to the exogenous latent variable are very close to their population counterparts. Thus, for all parameters belonging to the exogenous variable, estimates adhere to Proposition 3. This is because this part of the model is not affected by the misspecification.

With regard to the misspecified part of the model, Table 7 reveals that these estimates deviate considerably from the limit estimates, demonstrating the vanishing of the relation stated in Proposition 3. For instance, the limit estimate of the regression coefficient is $\widehat{\gamma}_{11}^{(\mathrm{FM} 1)}=$ $\frac{4.000}{2.000} \cdot 1.000=2.000$, but the actual estimate is $\widehat{\gamma}_{11}^{(\mathrm{FM} 1)}=1.841$. The same holds true for the limit of the residual variance of $\eta_{1}$, it should be $\widehat{\psi}_{11}^{(\text {FM1 })}=4.000^{2} \cdot 2.000=32.000$, but the actual estimate is $\widehat{\psi}_{11}^{(\mathrm{FM} 1)}=24.977$. Additionally, estimated manifest error variances deviate largely from their population quantities. However, the results in Table 7 demonstrate that estimated manifest residual errors do not depend on the scaling method, even for the misspecified part of the model.

\section{Wald Tests for Significance}

We will now consider how scaling methods affect standard errors, $Z$ values, and $p$ values of parameters. As we have seen, parameter estimates take different values under different scaling methods, and it is rather obvious that their estimated standard errors are sensitive to the choice of scaling method, too. de Pijper and Saris (1982) provide an example where 
a specific estimated loading of interest depends on the scaling method employed, and they also conduct a Monte Carlo study in which Wald tests of that parameter's significance using different scaling methods disagree in more than $10 \%$ of the cases. Additionally, Gonzalez and Griffin (2001) show that $Z$ and $p$ values depend on the scaling method not only for loading parameters, but also when considering latent covariances. Below, we will exemplify that $Z$ and $p$ values of parameters generally indeed depend on the scaling method used, drawing on the results from Proposition 3 and Remark 10. In particular, we will contribute to the literature by examining the regression coefficient describing the relation between two latent variables. For this example, we draw on the mediation model depicted in Figure 5.

To start our considerations, it is useful to have a closer look at the principles underlying Wald tests: these are constructed by dividing an estimator by its estimated standard deviation, e.g., the Wald statistic for $\widehat{\gamma}_{11}$ in the mediation example is given as $Z_{\widehat{\gamma}_{11}}:=\frac{\widehat{\gamma}_{11}}{\operatorname{se}\left(\widehat{\gamma}_{11}\right)}$, where $\widehat{\gamma}_{11}$ denotes an estimator for the regression coefficient, while se $\left(\widehat{\gamma}_{11}\right)$ denotes an estimate for $\widehat{\gamma}_{11}$ 's standard deviation. This Wald test statistic is designed for testing the null hypothesis that the quantity estimated by $\widehat{\gamma}_{11}$ is equal to zero.

For the mediation example maintained in this paper, we have five different Wald test statistics for the estimated regression coefficient $\widehat{\gamma}_{11}$ between the exogenous latent variable $\xi_{1}$ and the endogenous latent variable $\eta_{1}: Z^{(\mathrm{S})}:=\frac{\widehat{\gamma}_{11}^{(S)}}{\operatorname{se}\left(\widehat{\gamma}_{11}^{(\mathrm{S})}\right)}$, with $(\mathrm{S}) \in\{\mathrm{FM} 1, \mathrm{FM} 2, \mathrm{FM} 3, \mathrm{EC}, \mathrm{FF}\}$. As the limit estimates of $\widehat{\gamma}_{11}^{(\mathrm{FM} 1)}, \widehat{\gamma}_{11}^{(\mathrm{FM} 2)}, \widehat{\gamma}_{11}^{\mathrm{FM} 3)}, \widehat{\gamma}_{11}^{(\mathrm{FF})}$, and $\widehat{\gamma}_{11}^{(\mathrm{EC})}$ are $\frac{\lambda_{y, 11}^{(\mathrm{pop})}}{\lambda_{x, 11}^{(\mathrm{pop})}} \cdot \gamma_{11}^{(\mathrm{pop})}, \frac{\lambda_{y, 21}^{(\mathrm{pop})}}{\lambda_{x, 21}^{(\mathrm{pop})}} \cdot \gamma_{11}^{(\mathrm{pop})}$, $\frac{\lambda_{y, 31}^{(\mathrm{pop})}}{\lambda_{x, 31}^{(\mathrm{pop})}} \cdot \gamma_{11}^{(\mathrm{pop})}, \frac{\mathrm{A}\left(\lambda_{y, \bullet 1}^{(\mathrm{pop})}\right)}{\mathrm{A}\left(\lambda_{x, \bullet 1}^{(\mathrm{pop})}\right)} \cdot \gamma_{11}^{(\mathrm{pop})}$, and $\frac{\sqrt{\phi_{11}^{(\mathrm{pop})}}}{\sqrt{\psi_{11}^{(\mathrm{pop})}}} \cdot \gamma_{11}^{(\mathrm{pop})}$, respectively, the corresponding Wald tests' null hypotheses are $H_{0}^{(\mathrm{FM} 1)}: \frac{\lambda_{y, 11}^{(\mathrm{pop})}}{\lambda_{x, 11}^{(\mathrm{pop})}} \cdot \gamma_{11}^{(\mathrm{pop})}=0, H_{0}^{(\mathrm{FM} 2)}: \frac{\lambda_{y, 21}^{(\mathrm{pop})}}{\lambda_{x, 21}^{(\mathrm{pop})}} \cdot \gamma_{11}^{(\mathrm{pop})}=0, H_{0}^{(\mathrm{FM} 3)}$ : $\frac{\lambda_{y, 21}^{(\mathrm{pop})}}{\lambda_{x, 21}^{(\mathrm{pop})}} \cdot \gamma_{11}^{(\mathrm{pop})}=0, H_{0}^{(\mathrm{EC})}: \frac{\mathrm{A}\left(\lambda_{y, \bullet 1}^{(\mathrm{pop})}\right)}{\mathrm{A}\left(\lambda_{x, \bullet 1}^{(\mathrm{pop})}\right)} \cdot \gamma_{11}^{(\mathrm{pop})}=0$, and $H_{0}^{(\mathrm{FF})}: \frac{\sqrt{\phi_{11}^{(\mathrm{pop})}}}{\sqrt{\psi_{11}^{(\mathrm{pop})}}} \cdot \gamma_{11}^{(\mathrm{pop})}=0$. Fortunately, under the reasonable assumption that the involved population quantities $\frac{\lambda_{y, 11}^{(\text {pop })}}{\lambda_{x, 11}^{(\text {pop })}}$, $\frac{\lambda_{y, 21}^{(\mathrm{pop})}}{\lambda_{x, 21}^{(\mathrm{pop})}}, \frac{\lambda_{y, 31}^{(\mathrm{pop})}}{\lambda_{x, 31}^{(\mathrm{pop})}}, \frac{\mathrm{A}\left(\lambda_{y, \bullet 1}^{(\mathrm{pop})}\right)}{\mathrm{A}\left(\lambda_{x, \bullet 1}^{(\mathrm{pop})}\right)}$, and $\frac{\sqrt{\phi_{11}^{(\mathrm{pop})}}}{\sqrt{\psi_{11}^{(\mathrm{pop})}}}$ are all different from zero, these null hypotheses are all equivalent to $\gamma_{11}^{(\mathrm{pop})}=0$. Thus, the Wald test statistics $Z^{(\mathrm{FM} 1)}, Z^{(\mathrm{FM} 2)}, Z^{(\mathrm{FM} 3)}, Z^{(\mathrm{EC})}$, and $Z^{(\mathrm{FF})}$ all test the intended null hypothesis of a vanishing population regression coefficient, 
$H_{0}: \gamma_{11}^{(\text {pop })}=0$, against the alternative $H_{1}: \gamma_{11}^{(\text {pop })} \neq 0 .{ }^{23}$ Analogous considerations apply to the estimated regression coefficients $\widehat{\gamma}_{21}$ and $\widehat{\beta}_{21}$ and will therefore not be outlined here.

Note that according to Proposition 2, the estimated regression coefficient $\widehat{\gamma}_{11}$ under a given scaling method can be converted into the estimated regression coefficient $\widehat{\widehat{\gamma}}_{11}$ under another scaling method. Assuming that we estimate the model using FM1 scaling, the formulas in Proposition 2 indicate that the estimated regression coefficient is given by $\widehat{\gamma}_{11}^{(\mathrm{FM} 2)}=\frac{\widehat{\lambda}_{y, 21}^{(\mathrm{FM} 1)}}{\widehat{\lambda}_{x, 21}^{(\mathrm{FM} 1)}}$. $\widehat{\gamma}_{11}^{(\mathrm{FM} 1)}$ when FM2 scaling is used, by $\widehat{\gamma}_{11}^{(\mathrm{FM} 3)}=\frac{\widehat{\lambda}_{y, 31}^{(\mathrm{FM} 1)}}{\widehat{\lambda}_{x, 31}^{(\mathrm{FM} 1)}} \cdot \widehat{\gamma}_{11}^{(\mathrm{FM} 1)}$ when FM3 scaling is used, by $\widehat{\gamma}_{11}^{(\mathrm{EC})}=\frac{\mathrm{A}\left(\widehat{\lambda}_{y, \bullet j}^{(\mathrm{FM} 1)}\right)}{\mathrm{A}\left(\widehat{\lambda}_{x, \bullet i}^{(\mathrm{FM} 1)}\right)} \cdot \widehat{\gamma}_{11}^{(\mathrm{FM} 1)}$ when EC scaling is used, and by $\widehat{\gamma}_{11}^{(\mathrm{FF})}=\frac{\sqrt{\widehat{\phi}_{11}^{(\mathrm{FM} 1)}}}{\sqrt{\widehat{\psi}_{i i}^{(\mathrm{FM} 1)}}} \cdot \widehat{\gamma}_{11}^{(\mathrm{FM} 1)}$ when FF scaling is used. ${ }^{24}$ A common feature of these formulas is that they are of the form $F \cdot \widehat{\gamma}_{11}^{(\text {FM1) }}$, in which $F$ denotes the term appearing in front of the estimated regression coefficients under FM1 in the above equations.

Therefore, the corresponding Wald statistic $Z^{(\mathrm{S})}$ can be written as

$$
Z^{(\mathrm{S})}=\frac{F^{(\mathrm{S})} \cdot \widehat{\gamma}_{11}^{(\mathrm{FM} 1)}}{\operatorname{se}\left(F^{(\mathrm{S})} \cdot \widehat{\gamma}_{11}^{(\mathrm{FM} 1)}\right)}=\frac{\widehat{\gamma}_{11}^{(\mathrm{FM} 1)}}{\operatorname{se}\left(\widehat{\gamma}_{11}^{(\mathrm{FM} 1)}\right)} \frac{F^{(\mathrm{S})} \cdot \operatorname{se}\left(\widehat{\gamma}_{11}^{(\mathrm{FM} 1)}\right)}{\operatorname{se}\left(F^{(\mathrm{S})} \cdot \widehat{\gamma}_{11}^{(\mathrm{FM} 1)}\right)}=Z^{(\mathrm{FM} 1)} \cdot R^{(\mathrm{S})}
$$

where $R^{(\mathrm{S})}:=\frac{F^{(\mathrm{S})} \cdot \operatorname{se}\left(\widehat{\gamma}_{11}^{(\mathrm{FM} 1)}\right)}{\operatorname{se}\left(F^{(\mathrm{S})} \cdot \widehat{\gamma}_{11}^{(\mathrm{FM} 1)}\right)}=\frac{Z^{(\mathrm{S})}}{Z^{(\mathrm{FM} 1)}}$ denotes the ratio of the two Wald statistics.

If $R^{(\mathrm{S})}$ was unity, then the Wald test statistics $Z^{(\mathrm{S})}$ and $Z^{(\mathrm{FM} 1)}$ would coincide. $R^{(\mathrm{S})}$ equaling unity is obviously equivalent to the condition

$$
\text { se }\left(F^{(\mathrm{S})} \cdot \widehat{\gamma}_{11}^{(\mathrm{FM} 1)}\right)=F^{(\mathrm{S})} \cdot \operatorname{se}\left(\widehat{\gamma}_{11}^{(\mathrm{FM} 1)}\right)
$$

which in general does not hold true, because $F^{(\mathrm{S})}$ is not deterministic, but a random variable. ${ }^{25}$ Again, analogous considerations apply to the estimated regression coefficients $\widehat{\gamma}_{21}$ and $\widehat{\beta}_{21}$.

In order to study potential differences between the Wald tests, we conducted Monte Carlo studies, each with 10,000 repetitions, drawing on the mediation example displayed in Figure 5. First, we investigated potential differences between the Wald test under the alternative

\footnotetext{
${ }^{23}$ Please note that this argumentation only applies in case of the null hypothesis $H_{0}: \gamma_{11}^{(\mathrm{pop})}=0$, it does not apply in the case of the null hypothesis $H_{0}: \gamma_{11}^{(\mathrm{pop})}=c, c \in \mathbb{R} \backslash 0$.

${ }^{24}$ Analogous considerations can be done for all possible conversions.

${ }^{25}$ If $F^{(\mathrm{S})}$ was a non-negative constant, Equation (14) would be fulfilled.
} 
hypothesis, i.e., we simulated data under the assumption that the regression parameters $\gamma_{11}$, $\gamma_{21}$, and $\beta_{21}$ equal their population value as given in Figure 5. We tested three different null hypotheses, namely $\gamma_{11}=0, \gamma_{21}=0$, and $\beta_{21}=0$, which were all tested using five different scaling methods, FM1, FM2, FM3, EC, and FF, and four different sample sizes, 250, 500, 750, and 1000. Figure 7 shows box plots of the corresponding Wald statistics, together with dashed lines in different colors. These lines indicate the $\frac{\alpha}{2}$ and $1-\frac{\alpha}{2}$ quantiles of a standard normal distribution for $\alpha \in\{0.1,0.05,0.01,0.001\}$. Thus, for instance, a $\mathrm{Z}$ value falling between the inner two, orange colored dashed lines, indicates that the corresponding null hypothesis would not have been rejected at the $10 \%$ level, while a $\mathrm{Z}$ value beyond the blue dashed lines indicates a test statistic belonging to a test which rejects the null hypothesis even at the $1 \%$ level.

Figure 7 shows that the scaling method indeed has an influence on the Wald statistics and that the power to detect whether regression coefficients are different from zero depends on the scaling method employed. In particular, especially for small sample sizes and the tests for $\gamma_{11}$ and $\gamma_{21}$, it is easy to see that at common significance levels, power is at its lowest when FM2 is used. This result is most probably driven by the fact that for all latent variables, their second indicators are the ones loading lowest, highlighting the common wisdom not to choose indicators which load poorly on the corresponding latent variables. Somewhat more surprising is the very good performance of EC, which one might expect to be a kind of mixture or compromise of FM1-FM3, however, it does never perform worse than any of FM1, FM2, and FM3.

In order to also investigate the Wald statistics under the null hypothesis, we varied the simulation design and changed the value of $\gamma_{21}$ from .5 to 0 . The corresponding results are displayed in Figure 8. While, as expected, the results for the tests on $\gamma_{11}$ and $\beta_{21}$ stay more or less unchanged, the distribution of the Wald statistics for $\gamma_{21}$ is centered around zero and most results fall well within the bands defined by the quantiles, thus leading to (correctly) accepting the null hypothesis $\gamma_{21}=0$.

Overall, we can conclude that the results of Wald tests on parameters' significance in general depend on the scaling method, with the discrepancies between test results being minor when the null hypothesis is actually true. Under the alternative, however, some scaling methods may lead to much lower power than others. While it would be interesting to investigate which scaling method should be used under which circumstances, we leave this question to further research. 


\section{Conclusion and Remarks}

In this contribution, we provided a mathematical foundation of the three most common scaling methods applied in structural equation models with latent variables. We also provided a formal account of the possibility to convert estimated parameters between scaling methods without the need to re-estimate the model. We showed how estimated parameters relate to the population parameters. In particular, population parameters were shown not to be a specific numbers that is connected to a specific estimates. Instead, the same population parameter may have distinct quantities that are related to their estimates in non-unique ways. Using some rather basic examples, we to demonstrated the consequences of our propositions. It was shown that all of this has a common mathematical foundation. In the following, before making some remarks about the applications of our framework developed in this paper, we provide some critical remarks about the assumptions we made.

First of all, we introduced Assumption 1 which states that all latent variables need to be scaled. As mentioned above, this assumption holds true when there are no non-standard restrictions. Under this assumption, the entries in the diagonal matrices $\boldsymbol{D}_{\xi}$ and $\boldsymbol{D}_{\eta}$ used for rescaling are "free" in the sense that they can be set according to the scaling method used. A non-standard restriction would be, e.g., if the regression coefficient in our regression model in Figure 5 was fixed to some specific value $c$. In this case, the regression coefficient would no longer be estimated, estimation would instead be carried out under the constraint that $\widehat{\gamma}_{11}=c$. But this restriction would tie the scale of the latent endogenous variable $\eta_{1}$ to the scale of the latent exogenous variable $\xi_{1}$, implying that if one of these scales changes, then the other has to change accordingly. For instance, when the scale of $\xi_{1}$ were to be changed by the factor 10 , then the scale of $\eta_{1}$ has to change by the factor 10 , too, in order for the regression coefficient to stay unaffected.

When developing the mathematical foundation, we also built on Assumption 2 which states that all latent variables in a SEM are scaled using the same scaling method. As already mentioned at the end of section 2.1, this is certainly not a necessary assumption in our proposed framework, it was introduced to facilitate our considerations. Using a different scaling method for each latent variable in a SEM is indeed possible and can simply be modeled by using appropriate entries in the diagonal matrices $\boldsymbol{D}_{\xi}$ and $\boldsymbol{D}_{\eta}$. This refers to both the use of the diagonal matrices for conversion and for inferring the interpretation of the estimated parameters.

Assumption 2 is also the foundation for Table 4. Additionally, the interpretation rules in this table also rest on the assumption that all latent variables are scaled using the same scaling method. In the rare case of latent variables being scaled by more than one scaling 
method, the interpretation for such a particular combination might be derived by adapting the reasoning demonstrated in section 3.2, accordingly.

Finally, we also employed Assumption 3: The model under consideration is correctly specified, i.e., the specified model matches with population's dgp. This reflects a realist view of SEMs, which in the context of psychometric models has been discussed extensively by Borsboom (2005). In the broader contexts of SEMs, the match between dgp and the specified models means that there is a true theory about how the observable data came into existence. This view underpins the role of prior knowledge in a domain in the constructions of SEMs above stated. Thus, Assumption 3 can be read as "a model is correctly specified, in the sense that all causally relevant variables have been included and all irrelevant ones excluded" (Pratschke, 2003, p. 30) and that the directions of the effects are in accordance with theory. However, as there is (the possibility of) an underdetermination of theory by evidence, such theories can always be questioned and there may be misspecifications. As we have seen, when Assumption 3 is violated, i.e., the model is misspecified, issues arise regarding the estimated parameters' interpretation. Although there may be parameters that are not affected by the misspecification, in practice it is impossible to determine which part of the model may be affected and which may not. Therefore, researchers should carefully consider whether the available evidence supports their models or not. Moreover, there is always the possibility of equivalent models that cannot be told apart empirically (cf., Hershberger \& Marcoulides, 2013). For instance, changing the direction of the arrow in the regression example would not alter model fit. In this case, only substantial information from outside statistics may reveal the correct causal direction.

However, it is in practice, it is almost impossible to have full prior knowledge about the dgp. However, the results from Proposition 3 can be used in simulation studies in which the dgp is known and the limit estimates can be easily computed.

As a practical application, we studied the behavior of the Wald test under different scaling methods. Proposition 2 in combination with Proposition 3 revealed that the Wald test for a single parameter depends on the scaling method employed. With respect to Wald tests for the significance of regression parameters, we found that all scaling methods can be used. While these methods all lead to an asymptotic standard normal distribution of the test statistic under the null hypothesis of a vanishing regression coefficient, different scaling methods generally lead to different power for detecting a non-zero regression coefficient. Especially when researchers conducting such a Wald test obtain a $p$-value close to the chosen threshold, they might want to try other scaling methods in order to get more information on whether the corresponding regression coefficient is significantly different from zero. Moreover, as already noted by Gonzalez and Griffin (2001), testing whether a parameter differs from 0 is also pos- 
sible by means of a likelihood ratio test, which in this way is a supplement to the Wald test for researchers (see Gonzalez \& Griffin, 2001, p. 267 for details).

Another possible application of our conversion formulas would be to estimate the parameters $\hat{\theta}$ of a model under a certain scaling method and calculate the estimated parameters $\widehat{\hat{\theta}}$ and standard errors under any other scaling method. To this end, one could consider the conversion from $\widehat{\theta}$ to $\widehat{\hat{\theta}}$ as a vector valued function, whose components are the conversion rules given in Proposition 2. Using the estimated covariance matrix of the estimated parameter vector $\widehat{\theta}$, which is usually a result of the estimation process, the standard errors of the parameters $\widehat{\hat{\theta}}$ can be calculated by means of the delta method (Gonzalez \& Griffin, 2001). This procedure is implemented in modern SEM software, e.g., in lavaan, where the conversion and calculation of the standard errors can be done by means of defined parameters (which contain the conversion formulas). The same is also possible in Mplus and other SEM software.

Moreover, the conversion formulas provided in this paper make it easy to compare the results of studies that employed different scaling methods. Assuming that the structural equation models to be compared have the same structure, the results from different studies can be made comparable by converting parameters to a common metric implied by a specific scaling method.

Finally, we want to discuss how the three common scaling methods relate to the scaling approach proposed by Hayduk and Littvay (2012, p. 1). According to these authors, "[s]tructural equation modeling developed as a statistical melding of path analysis and factor analysis that obscured a fundamental tension between a factor preference for multiple indicators and path modeling's openness to fewer indicators" As a consequence, Hayduk and Littvay advocated the use of very few, or even only a single indicator per latent variable. Additionally, they proposed that the best indicator's loading should be fixed to unity, while restricting the corresponding manifest residual error variance to some pre-specified value. The latter serves to lend meaning to the latent variable, because the restriction on the manifest residual error variance is testable. Hayduk and Littvay argue that researchers should not only make assertions of the presence or absence of relations between latent variables, but also assertions about manifest residual errors, because these reflect a kind of theory assertion as well (see also Hayduk, 1988, pp. 118-125). From a mathematical perspective, this kind of scaling approach is a special case of FM scaling, accompanied by fixing the best indicator's residual variance to some predetermined value. As our results indeed hold for models with restricted residual (co-)variances (as outlined in the text following Assumption 2), the mathematical apparatus developed for the common scaling methods also applies to the scaling approach of Hayduk and Littvay (2012). 
However, one restriction has to be mentioned: if a manifest residual error variance is fixed and the fixed value does not correspond to its population quantity, than this is a misspecification, with the consequence that the manifest residual error variance would no longer be estimated as its population counterpart (as outlined in Remark 8). Additionally, depending on the estimation method used, there is the possibility that such a misspecification would also affect the estimation of all other parameters.

To conclude, the goal of this paper was to answer the question of how to interpret estimated parameters in structural equation models with latent variables and to explain why it is possible to convert parameter estimates obtained under one scaling method to those obtained under another scaling method. The answer to this question revealed that both the conversion of estimated parameters and the relation of estimated parameters and population parameters have the same mathematical structure. We found that both the interpretation of estimated parameters and the power of Wald tests for significance of parameters may depend on the scaling method used. Regarding interpretation, we found that some parameters are easy to interpret, while other parameter's interpretation is more intricate. For instance, estimated loadings are rather straightforward to interpret, and the different interpretations may be useful in psychometric studies (see the examples provided in this contribution and in Klößner \& Klopp, 2019). In particular, the interpretation of estimated factor loadings and estimated regression coefficients is always in the scale set by the respective scaling method. Furthermore, estimated latent (residual) (co-)variances are harder to interpret, expect for fixed factor scaling when these parameters bear some resemblance to correlations. Overall, all estimated parameters have one property in common: their interpretation involves some algebraic transformations of population parameters. Additionally, the numerical value of an estimated regression coefficient depends on the scaling method and, as shown at the end of section 3.2, there may be many different population quantities that yield the same estimated regression coefficient. Consequently, the question whether a population regression coefficient equals a given non-zero number cannot be answered empirically. In other words, the hypothesis that the population regression coefficient equals a given non-zero number is not identified. This also applies to other parameters except the manifest residual variances which do not depend on the scaling method (see Remark 1).

To finish, we would like to mention some exemplary settings in which the results from this contribution are relevant. The first of these settings is the one considered by Bielby (1986) who noted that the estimated regression coefficient deviates from its population value when using FM scaling in a situation where the referent indicator's population loading deviates from 1. Bielby further noted that this may hamper the comparison of estimated regression coefficients between two groups (cf., Guenole \& Brown, 2014). In general, even if the population regression 
coefficients are equal between two groups, their estimates may differ depending on the scaling method and the estimates may be incomparable. ${ }^{26}$ Similar considerations certainly apply to other model parameters and also to longitudinal settings e.g., Klößner and Klopp (2019). Finally, a meta analysis is a setting in which the comparison of parameters matters. Here, researchers should be aware that when estimated parameters are to be compared, they first must be made comparable in order to account for the particular methods of scaling used in the different analyses. To this end, the rules developed above for converting estimated parameters from one scaling method to another are a valuable tool.

In conclusion, applied researchers should always bear in mind that in SEMs with latent variables most estimated parameters do not directly represent population quantities. Instead, they estimate algebraic transformations of population parameters, affecting their interpretation and the power of significance tests. Therefore, it should be common practice in reports to clearly state which scaling method was used.

\section{Acknowledgements}

The authors are grateful for valuable remarks from Sandra Baar, Martin Becker, and Mireille Soliman. We thank Sina Wilhelm for her help in preparing the manuscript. Finally, our thanks also go to Martin Klein, the first author's lumberjack colleague.

\section{References}

Bartholomew, D. J., Knott, M., \& Moustaki, I. (2011). Latent variable models and factor analysis: a unified approach. Oxford: Wiley-Blackwell.

Bielby, W. T. (1986). Arbitrary metrics in multiple-indicator models of latent variables. Sociological Methods \& Research, 15(1-2), 3-23.

Bollen, K. A. (1989). Structural equations with latent variables. New York: Wiley.

Borsboom, D. (2005). Measuring the mind: Conceptual issues in contemporary psychometrics. Cambridge: Cambridge University Press.

de Pijper, W. M., \& Saris, W. E. (1982). The effect of identification restrictions on the test statistics in latent variable models. In K. Jöreskog \& H. Wold (Eds.), Systems under indirect observation (p. 175-182). Amsterdam: North-Holland.

Gonzalez, R., \& Griffin, D. (2001). Testing parameters in structural equation modeling: Every "One" matters. Psychological Methods, 6(3), 258-269.

\footnotetext{
${ }^{26}$ However, the explained variance $R^{2}$ is invariant towards the scaling method, see the supplemental material.
} 
Grace, J. (2006). Structural equation modeling and natural systems. Cambridge: Cambridge University Press.

Guenole, N., \& Brown, A. (2014). The consequences of ignoring measurement invariance for path coefficients in structural equation models. Frontiers in Psychology, 5, 980.

Hayduk, L. A. (1988). Structural equation modeling with LISREL: Essentials and advances (3. print. ed.). Baltimore: Johns Hopkins Univ. Pr.

Hayduk, L. A., \& Littvay, L. (2012). Should researchers use single indicators, best indicators, or multiple indicators in structural equation models? BMC Medical Research Methodology, 12(159).

Hershberger, S., \& Marcoulides, G. (2013). The problem of equivalent structural models. In G. Hancock \& R. Mueller (Eds.), Structural equation modeling: A second course (p. 3-39). Amsterdam: Information Age Publishing.

Jöreskog, K. (1978). Structural analysis of covariance and correlation matrices. Psychometrika, 43, 443-477.

Kaplan, D. W. (2000). Structural equation modeling: Foundations and extensions. Los Angeles: Sage.

Kline, R. B. (2016). Principles and practice of structural equation modeling (Fourth ed.). New York: The Guilford Press.

Klößner, S., \& Klopp, E. (2019). Explaining constraint interaction: How to interpret estimated model parameters under alternative scaling methods. Structural Equation Modeling: A Multidisciplinary Journal, 26(1), 143-155.

Little, T. D., Slegers, D. W., \& Card, N. A. (2006). A non-arbitrary method of identifying and scaling latent variables in sem and macs models. Structural Equation Modeling: A Multidisciplinary Journal, 13(1), 59-72.

Mulaik, S. A. (2010). Foundatons of factor analysis. Boca Raton: CRC Press.

Newsom, J. T. (2015). Longitudinal structural equation modeling: A comprehensive introduction. New York: Routledge.

Pearl, J. (2009). Causality: models, reasoning, and inference (2. ed.). Cambridge: Cambridge University Press.

Pratschke, J. (2003). Realistic models? Critical realism and statistical models in the social sciences. Philosophica, 71, 13-38.

R Core Team. (2018). R: A language and environment for statistical computing [Computer software manual]. Vienna, Austria. Retrieved from https://www.R-project.org/

Rigdon, E. (1995). A necessary and sufficient identification rule for structural models estimated in practice. Multivariate Behavioral Research, 30, 359-383.

Rosseel, Y. (2012). lavaan: An R package for structural equation modeling. Journal of 
Statistical Software, 48(2), 1-36. Retrieved from http://www.jstatsoft.org/v48/ i02/

SanMartin, E., Gonzalez, J., \& Tuerlinckx, F. (2015). On the Unidentifiablility of the FixedEffects 3PL Model. Psychometrika, 80, 450-467.

Schweizer, K. (2011). Scaling variances of latent variables by standardizing loadings: applications to working memory and the position effect. Multivariate Behavioral Research, $46,938-955$.

Schweizer, K., \& Troche, S. (2019). The EV scaling method for variances of latent variables. Methodology, 15, 175-184.

Schweizer, K., Troche, S., \& DiStefano, C. (2019). Scaling the variance of a latent variable while assuring constancy of the model. Frontiers in Psychology, 10, 887.

Shipley, B. (2004). Cause and correlation in biology. Cambridge: Cambridge University Press.

Skrondal, A., \& Rabe-Hesketh, S. (2004). Generalized latent variable modeling: multilevel, longitudinal, and structural equation models. Boca Raton: Chapman \& Hall/CRC.

Steiger, J. H. (2002). When constraints interact: a caution about reference variables, identification constraints, and scale dependencies in structural equation modeling. Psychological Methods, 7(2), 210-227.

von Oertzen, T. (2010). Power equivalence in structural equation modelling. British Journal of Mathematical and Statistical Psychology, 63, 257-272.

Wright, S. (1923). The theory of path coefficients: A reply to nile's criticism. Genetics, 8, 239-255.

Yoon, M., \& Millsap, R. E. (2007). Detecting violations of factorial invariance using databased specification searches: A Monte Carlo study. Structural Equation Modeling: A Multidisciplinary Journal, 14 (3), 435-463. 


\section{A Mathematical Appendix}

The Mathematical Appendix contains some lemmata which are used in the proofs in the second section. Both lemmata also contains assertions that refer to the content of the supplementary material.

Lemma 1. If $\boldsymbol{B}$ is a quadratic matrix such that $(\boldsymbol{I}-\boldsymbol{B})^{-1}$ exists and $\boldsymbol{D}$ be a non-singular diagonal matrix of the same dimension as $\boldsymbol{B}$, then $\boldsymbol{D}^{-1}(\boldsymbol{I}-\boldsymbol{B})^{-1} \boldsymbol{D}=\left(\boldsymbol{I}-\boldsymbol{D}^{-1} \boldsymbol{B} \boldsymbol{D}\right)^{-1}$.

Proof. The proof consists of simple linear algebra:

$\boldsymbol{D}^{-1}(\boldsymbol{I}-\boldsymbol{B})^{-1} \boldsymbol{D}=\left(\boldsymbol{D}^{-1}(\boldsymbol{I}-\boldsymbol{B}) \boldsymbol{D}\right)^{-1}=\left(\boldsymbol{D}^{-1} \boldsymbol{D}-\boldsymbol{D}^{-1} \boldsymbol{B} \boldsymbol{D}\right)^{-1}=\left(\boldsymbol{I}-\boldsymbol{D}^{-1} \boldsymbol{B} \boldsymbol{D}\right)^{-1}$.

Lemma 2. If $\theta=\left(\boldsymbol{\Lambda}_{x}, \boldsymbol{\Lambda}_{y}, \boldsymbol{\Gamma}, \boldsymbol{B}, \boldsymbol{\Phi}, \boldsymbol{\Psi}, \boldsymbol{\Theta}_{\delta}, \boldsymbol{\Theta}_{\epsilon}\right)$ and $\widetilde{\theta}=\left(\widetilde{\boldsymbol{\Lambda}}_{x}, \widetilde{\boldsymbol{\Lambda}} y, \widetilde{\boldsymbol{\Gamma}}, \widetilde{\boldsymbol{B}}, \widetilde{\boldsymbol{\Phi}}, \widetilde{\boldsymbol{\Psi}}, \widetilde{\boldsymbol{\Theta}}_{\delta}, \widetilde{\boldsymbol{\Theta}}_{\epsilon}\right)$ are related via a scale of change, then we have:

1. $\widetilde{\boldsymbol{A}}=\boldsymbol{D}_{\eta}^{-1} \boldsymbol{A} \boldsymbol{D}_{\eta}$ for $\widetilde{\boldsymbol{A}}:=(\boldsymbol{I}-\widetilde{\boldsymbol{B}})^{-1}$,

2. $\widetilde{\boldsymbol{\Gamma}} \widetilde{\boldsymbol{\Phi}} \widetilde{\boldsymbol{\Gamma}}^{\top}=\boldsymbol{D}_{\eta}^{-1} \boldsymbol{\Gamma} \boldsymbol{\Phi} \boldsymbol{\Gamma}^{\top} \boldsymbol{D}_{\eta}^{-1}$,

3. $\widetilde{\boldsymbol{\Gamma}} \widetilde{\boldsymbol{\Phi}} \widetilde{\boldsymbol{\Gamma}}^{\top}+\widetilde{\boldsymbol{\Psi}}=\boldsymbol{D}_{\eta}^{-1}\left(\boldsymbol{\Gamma} \boldsymbol{\Phi} \boldsymbol{\Gamma}^{\top}+\boldsymbol{\Psi}\right) \boldsymbol{D}_{\eta}^{-1}$,

4. $\widetilde{\boldsymbol{A}}\left(\widetilde{\boldsymbol{\Gamma}} \widetilde{\boldsymbol{\Phi}} \widetilde{\Gamma}^{\top}+\widetilde{\boldsymbol{\Psi}}\right) \widetilde{\boldsymbol{A}}^{\top}=\boldsymbol{D}_{\eta}^{-1} \boldsymbol{A}\left(\boldsymbol{\Gamma} \boldsymbol{\Phi} \boldsymbol{\Gamma}^{\top}+\boldsymbol{\Psi}\right) \boldsymbol{A}^{\top} \boldsymbol{D}_{\eta}^{-1}$,

5. for all $j \in\{1, \ldots, m\}:\left(\widetilde{\boldsymbol{A}}\left(\widetilde{\boldsymbol{\Gamma}} \widetilde{\boldsymbol{\Phi}} \widetilde{\boldsymbol{\Gamma}}^{\top}+\widetilde{\boldsymbol{\Psi}}\right) \widetilde{\boldsymbol{A}}^{\top}\right)_{j j}=\frac{\left(\boldsymbol{A}\left(\boldsymbol{\Gamma} \boldsymbol{\Phi} \boldsymbol{\Gamma}^{\top}+\boldsymbol{\Psi}\right) \boldsymbol{A}^{\top}\right)_{j j}}{d_{\eta, j}^{2}}$,

6. $\widetilde{\boldsymbol{\Lambda}}_{x} \widetilde{\boldsymbol{\Phi}} \widetilde{\boldsymbol{\Lambda}}_{x}^{\top}=\boldsymbol{\Lambda}_{x} \boldsymbol{\Phi} \boldsymbol{\Lambda}_{x}^{\top}$ and $\widetilde{\boldsymbol{\Lambda}}_{x} \widetilde{\boldsymbol{\Phi}} \widetilde{\boldsymbol{\Lambda}}_{x}^{\top}+\widetilde{\boldsymbol{\Theta}}_{\delta}=\boldsymbol{\Lambda}_{x} \boldsymbol{\Phi} \boldsymbol{\Lambda}_{x}^{\top}+\boldsymbol{\Theta}_{\delta}$,

7. $\widetilde{\boldsymbol{\Lambda}}_{y} \widetilde{\boldsymbol{A}} \widetilde{\boldsymbol{\Gamma}} \widetilde{\boldsymbol{\Phi}} \widetilde{\boldsymbol{\Lambda}}_{x}^{\top}=\boldsymbol{\Lambda}_{y} \boldsymbol{A} \boldsymbol{\Gamma} \boldsymbol{\Phi} \boldsymbol{\Lambda}_{x}^{\top}$ and $\widetilde{\boldsymbol{\Lambda}}_{x} \widetilde{\boldsymbol{\Phi}} \widetilde{\boldsymbol{\Gamma}}^{\top} \widetilde{\boldsymbol{A}}^{\top} \widetilde{\boldsymbol{\Lambda}}_{y}^{\top}=\boldsymbol{\Lambda}_{x} \boldsymbol{\Phi} \boldsymbol{\Gamma}^{\top} \boldsymbol{A}^{\top} \boldsymbol{\Lambda}_{y}^{\top}$,

8. $\widetilde{\boldsymbol{\Lambda}}_{y} \widetilde{\boldsymbol{A}}\left(\widetilde{\boldsymbol{\Gamma}} \widetilde{\boldsymbol{\Phi}} \widetilde{\boldsymbol{\Gamma}}^{\top}+\widetilde{\boldsymbol{\Psi}}\right) \widetilde{\boldsymbol{A}}^{\top} \widetilde{\boldsymbol{\Lambda}}_{y}^{\top}=\boldsymbol{\Lambda}_{y} \boldsymbol{A}\left(\boldsymbol{\Gamma} \boldsymbol{\Phi} \boldsymbol{\Gamma}^{\top}+\boldsymbol{\Psi}\right) \boldsymbol{A}^{\top} \boldsymbol{\Lambda}_{y}^{\top}$ and $\widetilde{\boldsymbol{\Lambda}}_{y} \widetilde{\boldsymbol{A}}\left(\widetilde{\boldsymbol{\Gamma}} \widetilde{\boldsymbol{\Phi}} \widetilde{\boldsymbol{\Gamma}}^{\top}+\widetilde{\boldsymbol{\Psi}}\right) \widetilde{\boldsymbol{A}}^{\top} \widetilde{\boldsymbol{\Lambda}}_{y}^{\top}+\widetilde{\boldsymbol{\Theta}}_{\epsilon}=\boldsymbol{\Lambda}_{y} \boldsymbol{A}\left(\boldsymbol{\Gamma} \boldsymbol{\Phi} \boldsymbol{\Gamma}^{\top}+\boldsymbol{\Psi}\right) \boldsymbol{A}^{\top} \boldsymbol{\Lambda}_{y}^{\top}+\boldsymbol{\Theta}_{\epsilon}$,

9. for any positive integer $r$ and any sequence $j_{0}, \ldots, j_{r}$ in $\{1, \ldots, m\}$, we have: $\widetilde{\beta}_{j_{r}, j_{r-1}} \cdot \ldots \cdot \widetilde{\beta}_{j_{1}, j_{0}}=\frac{d_{\eta, j_{0}}}{d_{\eta, j_{r}}} \cdot \beta_{j_{r}, j_{r-1}} \cdot \ldots \cdot \beta_{j_{1}, j_{0}}$, and, for any $i \in\{1, \ldots, n\}$, $\widetilde{\beta}_{j_{r}, j_{r-1}} \cdot \ldots \cdot \widetilde{\beta}_{j_{1}, j_{0}} \cdot \widetilde{\gamma}_{j_{0}, i}=\frac{d_{\xi, i}}{d_{\eta, j_{r}}} \cdot \beta_{j_{r}, j_{r-1}} \cdot \ldots \cdot \beta_{j_{1}, j_{0}} \cdot \gamma_{j_{0}, i}$,

10. for any non-negative integer $r$ : $\widetilde{\boldsymbol{B}}^{r}=\boldsymbol{D}_{\eta}^{-1} \boldsymbol{B}^{r} \boldsymbol{D}_{\eta}$ and $\widetilde{\boldsymbol{B}}^{r} \widetilde{\boldsymbol{\Gamma}}=\boldsymbol{D}_{\eta}^{-1} \boldsymbol{B}^{r} \boldsymbol{\Gamma} \boldsymbol{D}_{\xi}$.

Proof. 1. This follows from $\widetilde{\boldsymbol{B}}=\boldsymbol{D}_{\eta}^{-1} \boldsymbol{B} \boldsymbol{D}_{\eta}$ by applying Lemma 1 to $\boldsymbol{B}$ and $\boldsymbol{D}_{\eta}$.

2. $\widetilde{\boldsymbol{\Gamma}} \widetilde{\boldsymbol{\Phi}} \widetilde{\boldsymbol{\Gamma}}^{\top}=\boldsymbol{D}_{\eta}^{-1} \boldsymbol{\Gamma} \boldsymbol{D}_{\xi} \boldsymbol{D}_{\xi}^{-1} \boldsymbol{\Phi} \boldsymbol{D}_{\xi}^{-1}\left(\boldsymbol{D}_{\eta}^{-1} \boldsymbol{\Gamma} \boldsymbol{D}_{\xi}\right)^{\top}=\boldsymbol{D}_{\eta}^{-1} \boldsymbol{\Gamma} \boldsymbol{\Phi} \boldsymbol{D}_{\xi}^{-1} \boldsymbol{D}_{\xi} \boldsymbol{\Gamma}^{\top} \boldsymbol{D}_{\eta}^{-1}=\boldsymbol{D}_{\eta}^{-1} \boldsymbol{\Gamma} \boldsymbol{\Phi} \boldsymbol{\Gamma}^{\top} \boldsymbol{D}_{\eta}^{-1}$. 
3. $\widetilde{\boldsymbol{\Gamma}} \widetilde{\boldsymbol{\Phi}} \widetilde{\boldsymbol{\Gamma}}^{\top}+\widetilde{\boldsymbol{\Psi}} \stackrel{2 .}{=} \boldsymbol{D}_{\eta}^{-1} \boldsymbol{\Gamma} \boldsymbol{\Phi} \boldsymbol{\Gamma}^{\top} \boldsymbol{D}_{\eta}^{-1}+\boldsymbol{D}_{\eta}^{-1} \boldsymbol{\Psi} \boldsymbol{D}_{\eta}^{-1}=\boldsymbol{D}_{\eta}^{-1}\left(\boldsymbol{\Gamma} \boldsymbol{\Phi} \boldsymbol{\Gamma}^{\top}+\boldsymbol{\Psi}\right) \boldsymbol{D}_{\eta}^{-1}$.

4. $\widetilde{\boldsymbol{A}}\left(\widetilde{\boldsymbol{\Gamma}} \widetilde{\boldsymbol{\Phi}} \widetilde{\boldsymbol{\Gamma}}^{\top}+\widetilde{\boldsymbol{\Psi}}\right) \widetilde{\boldsymbol{A}}^{\top} \stackrel{1,3.3 .}{=} \boldsymbol{D}_{\eta}^{-1} \boldsymbol{A} \boldsymbol{D}_{\eta} \boldsymbol{D}_{\eta}^{-1}\left(\boldsymbol{\Gamma} \boldsymbol{\Phi} \boldsymbol{\Gamma}^{\top}+\boldsymbol{\Psi}\right) \boldsymbol{D}_{\eta}^{-1}\left(\boldsymbol{D}_{\eta}^{-1} \boldsymbol{A} \boldsymbol{D}_{\eta}\right)^{\top}$ $=\boldsymbol{D}_{\eta}^{-1} \boldsymbol{A}\left(\boldsymbol{\Gamma} \boldsymbol{\Phi} \boldsymbol{\Gamma}^{\top}+\boldsymbol{\Psi}\right) \boldsymbol{D}_{\eta}^{-1} \boldsymbol{D}_{\eta} \boldsymbol{A}^{\top} \boldsymbol{D}_{\eta}^{-1}=\boldsymbol{D}_{\eta}^{-1} \boldsymbol{A}\left(\boldsymbol{\Gamma} \boldsymbol{\Phi} \boldsymbol{\Gamma}^{\top}+\boldsymbol{\Psi}\right) \boldsymbol{A}^{\top} \boldsymbol{D}_{\eta}^{-1}$.

5. This follows immediately from the previous assertion.

6. $\widetilde{\Lambda}_{x} \widetilde{\boldsymbol{\Phi}} \widetilde{\Lambda}_{x}^{\top}=\boldsymbol{\Lambda}_{x} \boldsymbol{D}_{\xi} \boldsymbol{D}_{\xi}^{-1} \boldsymbol{\Phi} \boldsymbol{D}_{\xi}^{-1}\left(\boldsymbol{\Lambda}_{x} \boldsymbol{D}_{\xi}\right)^{\top}=\boldsymbol{\Lambda}_{x} \boldsymbol{\Phi} \boldsymbol{D}_{\xi}^{-1} \boldsymbol{D}_{\xi} \boldsymbol{\Lambda}_{x}^{\top}=\boldsymbol{\Lambda}_{x} \boldsymbol{\Phi} \boldsymbol{\Lambda}_{x}^{\top}$, while the second assertion follows from $\widetilde{\Theta}_{\delta}=\Theta_{\delta}$.

7. $\widetilde{\boldsymbol{\Lambda}}_{y} \widetilde{\boldsymbol{A}} \widetilde{\boldsymbol{\Gamma}} \widetilde{\boldsymbol{\Phi}} \widetilde{\boldsymbol{\Lambda}}_{x}^{\top} \stackrel{1 .}{=} \boldsymbol{\Lambda}_{y} \boldsymbol{D}_{\eta} \boldsymbol{D}_{\eta}^{-1} \boldsymbol{A} \boldsymbol{D}_{\eta} \boldsymbol{D}_{\eta}^{-1} \boldsymbol{\Gamma} \boldsymbol{D}_{\xi} \boldsymbol{D}_{\xi}^{-1} \boldsymbol{\Phi} \boldsymbol{D}_{\xi}^{-1}\left(\boldsymbol{\Lambda}_{x} \boldsymbol{D}_{\xi}\right)^{\top}=\boldsymbol{\Lambda}_{y} \boldsymbol{A} \boldsymbol{\Gamma} \boldsymbol{\Phi} \boldsymbol{\Lambda}_{x}^{\top}$, while the second assertion follows by taking the transpose on both sides.

8. $\widetilde{\boldsymbol{\Lambda}}_{y} \widetilde{\boldsymbol{A}}\left(\widetilde{\boldsymbol{\Gamma}} \widetilde{\boldsymbol{\Phi}} \widetilde{\boldsymbol{\Gamma}}^{\top}+\widetilde{\boldsymbol{\Psi}}\right) \widetilde{\boldsymbol{A}}^{\top} \widetilde{\boldsymbol{\Lambda}}_{y}^{\top} \stackrel{1 ., 4 .}{=} \boldsymbol{\Lambda}_{y} \boldsymbol{D}_{\eta} \boldsymbol{D}_{\eta}^{-1} \boldsymbol{A}\left(\boldsymbol{\Gamma} \boldsymbol{\Phi} \boldsymbol{\Gamma}^{\top}+\boldsymbol{\Psi}\right) \boldsymbol{A}^{\top} \boldsymbol{D}_{\eta}^{-1}\left(\boldsymbol{\Lambda}_{y} \boldsymbol{D}_{\eta}\right)^{\top}$ $=\boldsymbol{\Lambda}_{y} \boldsymbol{A}\left(\boldsymbol{\Gamma} \boldsymbol{\Phi} \boldsymbol{\Gamma}^{\top}+\boldsymbol{\Psi}\right) \boldsymbol{A}^{\top} \boldsymbol{\Lambda}_{y}^{\top}$, while the second assertion follows from $\widetilde{\boldsymbol{\Theta}}_{\epsilon}=\boldsymbol{\Theta}_{\epsilon}$.

9. $\widetilde{\beta}_{j_{r}, j_{r-1}} \cdot \ldots \cdot \widetilde{\beta}_{j_{1}, j_{0}}=\frac{d_{\eta, j_{r-1}}}{d_{\eta, j_{r}}} \beta_{j_{r}, j_{r-1}} \cdot \ldots \cdot \frac{d_{\eta, j_{0}}}{d_{\eta, j_{1}}} \beta_{j_{1}, j_{0}}=\frac{d_{\eta, j_{0}}}{d_{\eta, j_{r}}} \cdot \beta_{j_{r}, j_{r-1}} \cdot \ldots \cdot \beta_{j_{1}, j_{0}}$, while the second assertion follows by employing $\widetilde{\gamma}_{j_{0} i}=\frac{d_{\xi, i}}{d_{\eta, j_{0}}} \gamma_{j_{0} i}$.

10. The first assertion follows easily from $\widetilde{\boldsymbol{B}}=\boldsymbol{D}_{\eta}^{-1} \boldsymbol{B}^{r} \boldsymbol{D}_{\eta}$ by induction on $r$, while the second assertion follows from the first one by inserting $\widetilde{\boldsymbol{\Gamma}}=\boldsymbol{D}_{\eta}^{-1} \boldsymbol{\Gamma} \boldsymbol{D}_{\xi}$.

\section{B Covariances matrices}

B.1: Sample covariance matrix for the regression example in Figure 2.

\begin{tabular}{rrrrr}
\hline & $x_{11}$ & $x_{21}$ & $y_{12}$ & $y_{22}$ \\
\hline$x_{11}$ & 1.1621 & & & \\
$x_{21}$ & 1.977 & 4.2903 & & \\
$y_{12}$ & 11.999 & 24.8417 & 212.0985 & \\
$y_{22}$ & 1.5428 & 3.1687 & 27.0653 & 3.6544 \\
\hline
\end{tabular}


B.2: Population covariance matrix for the regression example in Figure 2.

\begin{tabular}{rrrrr}
\hline & $x_{11}$ & $x_{21}$ & $y_{11}$ & $y_{21}$ \\
\hline$x_{11}$ & 1.200 & & & \\
$x_{12}$ & 2.000 & 4.200 & & \\
$y_{11}$ & 12.000 & 24.000 & 208.200 & \\
$y_{21}$ & 1.500 & 3.000 & 26.000 & 3.450 \\
\hline
\end{tabular}

B.3: Population covariance matrix for the mediation example in Figure 5.

\begin{tabular}{rrrrrrrrrr}
\hline & \multicolumn{1}{c}{$x_{1}$} & \multicolumn{1}{c}{$x_{2}$} & \multicolumn{1}{c}{$x_{3}$} & \multicolumn{1}{c}{$y_{1}$} & $y_{2}$ & $y_{3}$ & $y_{4}$ & $y_{5}$ & $y_{6}$ \\
\hline$x_{1}$ & 29.000 & & & & & & & & \\
$x_{2}$ & 2.000 & 26.000 & & & & & & & \\
$x_{3}$ & 4.000 & 2.000 & 29.000 & & & & & & \\
$y_{1}$ & 8.000 & 4.000 & 8.000 & 73.000 & & & & & \\
$y_{2}$ & 2.000 & 1.000 & 2.000 & 12.000 & 28.000 & & & & \\
$y_{3}$ & 10.000 & 5.000 & 10.000 & 60.000 & 15.000 & 100.000 & & & \\
$y_{4}$ & 30.000 & 15.000 & 30.000 & 156.000 & 39.000 & 195.000 & 682.000 & & \\
$y_{5}$ & 5.000 & 2.500 & 5.000 & 26.000 & 6.500 & 32.500 & 109.500 & 43.2500 & \\
$y_{6}$ & 15.000 & 7.500 & 15.000 & 78.000 & 19.500 & 97.500 & 328.500 & 54.750 & 189.250 \\
\hline
\end{tabular}




\section{Tables}

Table 1: Estimation results for the model in Figure 2.

\begin{tabular}{lrrrrc}
\hline Parameter & Pop. & FM1 & FM2 & EC & FF \\
\hline$\widehat{\lambda}_{x, 11}$ & 1.000 & 1.000 & 0.483 & 0.651 & 0.976 \\
$\widehat{\lambda}_{x, 21}$ & 2.000 & 2.070 & 1.000 & 1.349 & 2.021 \\
$\widehat{\lambda}_{y, 11}$ & 4.000 & 1.000 & 7.828 & 1.773 & 7.807 \\
$\widehat{\lambda}_{y, 21}$ & 0.500 & 0.128 & 1.000 & 0.227 & 0.997 \\
$\widehat{\gamma}_{11}$ & 3.000 & 12.565 & 0.775 & 4.616 & 1.571 \\
$\widehat{\phi}_{11}$ & 1.000 & 0.953 & 4.084 & 2.246 & 1.000 \\
$\widehat{\psi}_{11}$ & 4.000 & 60.942 & 0.995 & 19.377 & 1.000 \\
$\widehat{\theta}_{\delta, 11}$ & 0.200 & 0.207 & 0.207 & 0.207 & 0.207 \\
$\widehat{\theta}_{\delta, 21}$ & 0.200 & 0.198 & 0.198 & 0.198 & 0.198 \\
$\widehat{\theta}_{\epsilon, 11}$ & 0.200 & 0.220 & 0.220 & 0.220 & 0.220 \\
$\widehat{\theta}_{\epsilon, 21}$ & 0.200 & 0.197 & 0.197 & 0.197 & 0.197 \\
\hline
\end{tabular}


Table 2: Rules for converting parameter values into corresponding values for the fixed marker, fixed factor, and effects coding scaling methods.

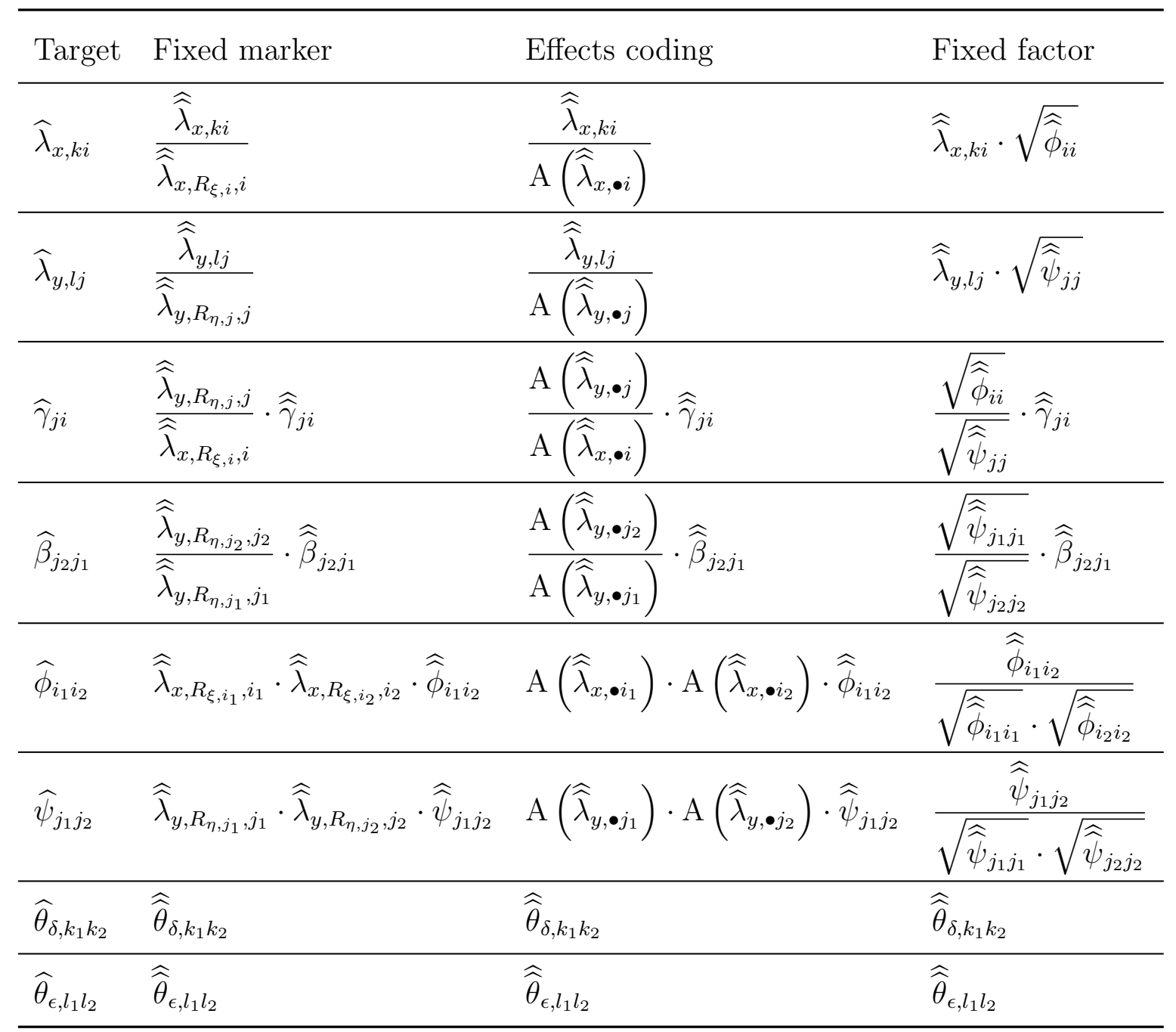

Note. The index R refers to the reference indicator in FM scaling according to Definition 3. 
Table 3: Quantities that are represented by the parameter estimates, for the FM, FF, and EC scaling methods.

\begin{tabular}{|c|c|c|c|}
\hline Parameter & Fixed marker & Effects coding & Fixed factor \\
\hline$\widehat{\lambda}_{x, k i}$ & $\frac{\lambda_{x, k i}^{(\mathrm{pop})}}{\lambda_{x, R_{\xi, i}, i}^{(\mathrm{pop})}}$ & $\frac{\lambda_{x, k i}^{(\mathrm{pop})}}{\mathrm{A}\left(\lambda_{x, \bullet i}^{(\mathrm{pop})}\right)}$ & $\lambda_{x, k i}^{(\mathrm{pop})} \cdot \sqrt{\phi_{i i}^{(\mathrm{pop})}}$ \\
\hline$\widehat{\lambda}_{y, l j}$ & $\frac{\lambda_{y, l j}^{(\mathrm{pop})}}{\lambda_{y, R_{\eta, j}, j}^{(\mathrm{pop})}}$ & $\frac{\lambda_{y, l j}^{(\mathrm{pop})}}{\mathrm{A}\left(\lambda_{y, \bullet j}^{(\mathrm{pop})}\right)}$ & $\lambda_{y, l j}^{(\mathrm{pop})} \cdot \sqrt{\psi_{j j}^{(\mathrm{pop})}}$ \\
\hline$\widehat{\gamma}_{j i}$ & $\frac{\lambda_{y, R_{\eta, j}, j}^{(\mathrm{pop})}}{\lambda_{x, R_{\xi, i}, i}^{(\mathrm{pop})}} \cdot \gamma_{j i}^{(\mathrm{pop})}$ & $\frac{\mathrm{A}\left(\lambda_{y, \bullet j}^{(\mathrm{pop})}\right)}{\mathrm{A}\left(\lambda_{x, \bullet i}^{(\mathrm{pop})}\right)} \cdot \gamma_{j i}^{(\mathrm{pop})}$ & $\frac{\sqrt{\phi_{i i}^{(\mathrm{pop})}}}{\sqrt{\psi_{j j}^{(\mathrm{pop})}}} \cdot \gamma_{j i}^{(\mathrm{pop})}$ \\
\hline$\widehat{\beta}_{j_{2} j_{1}}$ & $\frac{\lambda_{y, R_{\eta, j_{2}}, j_{2}}^{(\mathrm{pop})}}{\lambda_{y, R_{\eta, j_{1}}, j_{1}}^{(\mathrm{pop})}} \cdot \beta_{j_{2} j_{1}}^{(\mathrm{pop})}$ & $\frac{\mathrm{A}\left(\lambda_{y, \bullet j_{2}}^{(\mathrm{pop})}\right)}{\mathrm{A}\left(\lambda_{y, \bullet j_{1}}^{(\mathrm{pop})}\right)} \cdot \beta_{j_{2} j_{1}}^{(\mathrm{pop})}$ & $\frac{\sqrt{\psi_{j_{1} j_{1}}^{(\mathrm{pop})}}}{\sqrt{\psi_{j_{2} j_{2}}^{(\mathrm{pop})}}} \cdot \beta_{j_{2} j_{1}}^{(\mathrm{pop})}$ \\
\hline$\widehat{\phi}_{i_{1} i_{2}}$ & $\lambda_{x, R_{\xi, i_{1}, i_{1}}}^{(\mathrm{pop})} \cdot \lambda_{x, R_{\xi, i_{2}, i_{2}}}^{(\mathrm{pop})} \cdot \phi_{i_{1} i_{2}}^{(\mathrm{pop})}$ & $\mathrm{A}\left(\lambda_{x, \bullet i_{1}}^{(\mathrm{pop})}\right) \cdot \mathrm{A}\left(\lambda_{x, \bullet i_{2}}^{(\mathrm{pop})}\right) \cdot \phi_{i_{1} i_{2}}^{(\mathrm{pop})}$ & $\frac{\phi_{i_{1} i_{2}}^{(\mathrm{pop})}}{-}$ \\
\hline \multirow{2}{*}{$\widehat{\psi}_{j_{1} j_{2}}$} & \multirow{2}{*}{$\lambda_{y, R_{\eta, j_{1}}, j_{1}}^{(\mathrm{pop})} \cdot \lambda_{y, R_{\eta, j_{2}}, j_{2}}^{(\mathrm{pop})} \cdot \psi_{j_{1} j_{2}}^{(\mathrm{pop})}$} & \multirow{2}{*}{$\mathrm{A}\left(\lambda_{y, \bullet j_{1}}^{(\mathrm{pop})}\right) \cdot \mathrm{A}\left(\lambda_{y, \bullet j_{2}}^{(\mathrm{pop})}\right) \cdot \psi_{j_{1} j_{2}}^{(\mathrm{pop})}$} & $\psi_{j_{1} j_{2}}^{(\mathrm{pop})}$ \\
\hline & & & $\sqrt{\psi_{j_{1} j_{1}}^{(\mathrm{pop})}} \cdot \sqrt{\psi_{j_{2} j_{2}}^{(\mathrm{pop})}}$ \\
\hline$\widehat{\theta}_{\delta, k_{1} k_{2}}$ & $\theta_{\delta, k_{1} k_{2}}^{(\mathrm{pop})}$ & $\theta_{\delta, k_{1} k_{2}}^{(\mathrm{pop})}$ & $\theta_{\delta, k_{1} k_{2}}^{(\mathrm{pop})}$ \\
\hline$\widehat{\theta}_{\epsilon, l_{1} l_{2}}$ & $\theta_{\epsilon, l_{1} l_{2}}^{(\text {pop })}$ & $\theta_{\epsilon, l_{1} l_{2}}^{(\mathrm{pop})}$ & $\theta_{\epsilon, l_{1} l_{2}}^{(\mathrm{pop})}$ \\
\hline
\end{tabular}

Note. The index $\mathrm{R}$ refers to the reference indicator in FM scaling according to Definition 3. 
Table 4: Interpretation templates for the FM, FF, and EC scaling methods.

\begin{tabular}{|c|c|c|c|}
\hline Parameter & Fixed marker & Effects coding & Fixed factor \\
\hline$\widehat{\lambda}$ & $\begin{array}{l}\text { The respective indicator loads } \hat{\lambda} \text { times } \\
\text { as strongly on the latent variable as its } \\
\text { referent indicator does. }\end{array}$ & $\begin{array}{l}\text { The respective indicator loads } \widehat{\lambda} \text { times } \\
\text { as strongly on the latent variable as its } \\
\text { average indicator does. }\end{array}$ & $\begin{array}{l}\text { When the latent variable changes by } \\
\text { one standard deviation (exogenous } \\
\text { variables) or by one standard deviation } \\
\text { of its residual (endogenous variables), } \\
\text { then the indicator changes by } \widehat{\lambda} \text { units. }\end{array}$ \\
\hline$\widehat{\gamma}$ & $\begin{array}{l}\text { When the exogenous latent variable } \\
\text { changes in such a way that its referent } \\
\text { indicator changes by one unit, then the } \\
\text { endogenous latent variable changes in } \\
\text { such a way that its referent indicator } \\
\text { changes by } \widehat{\gamma} \text { units. }\end{array}$ & $\begin{array}{l}\text { When the exogenous latent variable } \\
\text { changes in such a way that its average } \\
\text { indicator changes by one unit, then the } \\
\text { endogenous latent variable changes in } \\
\text { such a way that its average indicator } \\
\text { changes by } \widehat{\gamma} \text { units. }\end{array}$ & $\begin{array}{l}\text { When the exogenous latent variable } \\
\text { changes by one standard deviation, } \\
\text { then the endogenous latent variable } \\
\text { changes by } \widehat{\gamma} \text { standard deviations of its } \\
\text { residual. }\end{array}$ \\
\hline$\widehat{\beta}$ & $\begin{array}{l}\text { When the initial endogenous latent } \\
\text { variable changes in such a way that its } \\
\text { referent indicator changes by one unit, } \\
\text { then the target latent variable changes } \\
\text { in such a way that its referent indicator } \\
\text { changes by } \widehat{\beta} \text { units. }\end{array}$ & $\begin{array}{l}\text { When the initial endogenous latent } \\
\text { variable changes in such a way that its } \\
\text { average indicator changes by one unit, } \\
\text { then the target latent variable changes } \\
\text { in such a way that its average indicator } \\
\text { changes by } \widehat{\beta} \text { units. }\end{array}$ & $\begin{array}{l}\text { When the initial endogenous latent } \\
\text { variable changes by one standard de- } \\
\text { viation of its residual, then the target } \\
\text { latent variable changes by } \widehat{\beta} \text { standard } \\
\text { deviations of its own residual. }\end{array}$ \\
\hline$\widehat{\phi}$ & $\begin{array}{l}\widehat{\psi} \text { estimates the amount of (co)variance } \\
\text { of (between) the latent variable's refer- } \\
\text { ent indicator(s) which is caused by the } \\
\text { latent variable(s). }\end{array}$ & $\begin{array}{l}\widehat{\psi} \text { estimates the amount of (co)variance } \\
\text { of (between) the latent variable's aver- } \\
\text { age indicator(s) which is caused by the } \\
\text { latent variable(s). }\end{array}$ & $\begin{array}{l}\text { Variances are fixed to unity and have } \\
\text { no interpretation. Covariances esti- } \\
\text { mate the correlation between the ex- } \\
\text { ogenous latent variables. }\end{array}$ \\
\hline$\widehat{\psi}$ & $\begin{array}{l}\widehat{\psi} \text { estimates the amount of (co)variance } \\
\text { of (between) the latent variable's refer- } \\
\text { ent indicator(s) which is caused by the } \\
\text { latent variable's residual(s). }\end{array}$ & $\begin{array}{l}\widehat{\psi} \text { estimates the amount of (co)variance } \\
\text { of (between) the latent variable's aver- } \\
\text { age indicator(s) which is caused by the } \\
\text { latent variable's residual(s). }\end{array}$ & $\begin{array}{l}\text { Variances are fixed to unity and have } \\
\text { no interpretation. Covariances esti- } \\
\text { mate the correlation between the en- } \\
\text { dogenous latent variables. }\end{array}$ \\
\hline
\end{tabular}


Table 5: Limit estimates of the parameters estimated for the model given in Figure 2.

\begin{tabular}{lrrrrr}
\hline Parameter & Pop. & \multicolumn{1}{c}{ FM1 } & FM2 & EC & FF \\
\hline$\lambda_{x, 11}$ & 1.000 & 1.000 & 0.500 & 0.667 & 1.000 \\
$\lambda_{x, 21}$ & 2.000 & 2.000 & 1.000 & 1.333 & 2.000 \\
$\lambda_{y, 11}$ & 4.000 & 1.000 & 8.000 & 1.778 & 8.000 \\
$\lambda_{y, 21}$ & 0.500 & 0.125 & 1.000 & 0.222 & 1.000 \\
$\gamma_{11}$ & 3.000 & 12.000 & 0.750 & 4.500 & 1.500 \\
$\phi_{11}$ & 1.000 & 1.000 & 4.000 & 2.250 & 1.000 \\
$\psi_{11}$ & 4.000 & 64.000 & 1.000 & 20.250 & 1.000 \\
$\theta_{\delta, 11}$ & 0.200 & 0.200 & 0.200 & 0.200 & 0.200 \\
$\theta_{\delta, 21}$ & 0.200 & 0.200 & 0.200 & 0.200 & 0.200 \\
$\theta_{\epsilon, 11}$ & 0.200 & 0.200 & 0.200 & 0.200 & 0.200 \\
$\theta_{\epsilon, 21}$ & 0.200 & 0.200 & 0.200 & 0.200 & 0.200 \\
\hline
\end{tabular}


Table 6: Conversions in the regression example.

\begin{tabular}{|c|c|c|c|}
\hline Parameter & FM1 & $\mathrm{EC}$ & $\mathrm{FF}$ \\
\hline$\widehat{\lambda}_{x, 11}$ & 1.000 & $\frac{1.000}{1.500}=0.667$ & $1.000 \cdot \sqrt{1.000}=1.000$ \\
\hline$\widehat{\lambda}_{x, 21}$ & 2.000 & $\frac{2.000}{1.500}=1.333$ & $2.000 \cdot \sqrt{1.000}=2.000$ \\
\hline$\widehat{\lambda}_{y, 11}$ & 1.000 & $\frac{1.000}{0.5625}=1.778$ & $1.000 \cdot \sqrt{64.000}=8.000$ \\
\hline$\widehat{\lambda}_{y, 21}$ & 0.125 & $\frac{0.125}{0.5625}=0.222$ & $0.125 \cdot \sqrt{64.000}=1.000$ \\
\hline$\widehat{\gamma}_{11}$ & 12.000 & $\frac{0.5625}{1.500} \cdot 12.000=4.500$ & $\frac{\sqrt{1.000}}{\sqrt{64.000}} \cdot 12.000=1.500$ \\
\hline$\widehat{\phi}_{11}$ & 1.000 & $1.500^{2} \cdot 1.000=2.250$ & $\frac{1.000}{\sqrt{1.000} \cdot \sqrt{1.000}}=1.000$ \\
\hline$\widehat{\psi}_{11}$ & 64.000 & $0.5625^{2} \cdot 64.000=20.250$ & $\frac{64.000}{\sqrt{64.000} \cdot \sqrt{64.000}}=1.000$ \\
\hline Parameter & $\mathrm{EC}$ & FM1 & $\mathrm{FF}$ \\
\hline$\widehat{\lambda}_{x, 11}$ & 0.667 & $\frac{0.667}{0.667}=1.000$ & $0.667 \cdot \sqrt{2.250}=1.000$ \\
\hline$\widehat{\lambda}_{x, 21}$ & 1.333 & $\frac{1.333}{0.667}=2.000$ & $1.333 \cdot \sqrt{2.250}=2.000$ \\
\hline$\widehat{\lambda}_{y, 11}$ & 1.778 & $\frac{1.778}{1.778}=1.000$ & $1.778 \cdot \sqrt{20.250}=8.000$ \\
\hline$\widehat{\lambda}_{y, 21}$ & 0.222 & $\frac{0.222}{1.778}=0.125$ & $0.222 \cdot \sqrt{20.250}=1.000$ \\
\hline$\widehat{\gamma}_{11}$ & 4.500 & $\frac{1.778}{0.667} \cdot 4.500=12.000$ & $\frac{\sqrt{2.250}}{\sqrt{20.250}} \cdot 4.500=1.500$ \\
\hline$\widehat{\phi}_{11}$ & 2.250 & $0.667^{2} \cdot 2.250=1.000$ & $\frac{2.250}{\sqrt{2.250} \cdot \sqrt{2.250}}=1.000$ \\
\hline$\widehat{\psi}_{11}$ & 20.250 & $1.778^{2} \cdot 20.250=64.000$ & $\frac{20.250}{\sqrt{20.250} \cdot \sqrt{20.250}}=1.000$ \\
\hline Parameter & $\mathrm{FF}$ & FM1 & $\mathrm{EC}$ \\
\hline$\widehat{\lambda}_{x, 11}$ & 1.000 & $\frac{1.000}{1.000}=1.000$ & $\frac{1.000}{1.500}=0.667$ \\
\hline$\widehat{\lambda}_{x, 21}$ & 2.000 & $\frac{2.000}{1.000}=1.000$ & $\frac{2.000}{1.500}=1.333$ \\
\hline$\widehat{\lambda}_{y, 11}$ & 8.000 & $\frac{8.000}{8.000}=1.000$ & $\frac{8.000}{4.500}=1.778$ \\
\hline$\widehat{\lambda}_{y, 21}$ & 1.000 & $\frac{1.000}{8.000}=0.125$ & $\frac{1.000}{4.500}=0.222$ \\
\hline$\widehat{\gamma}_{11}$ & 1.500 & $\frac{8.000}{1.000} \cdot 1.500=12.000$ & $\frac{4.500}{1.500} \cdot 1.500=4.500$ \\
\hline$\widehat{\phi}_{11}$ & 1.000 & $1.000^{2} \cdot 1.000=1.000$ & $1.500^{2} \cdot 1.000=2.250$ \\
\hline$\widehat{\psi}_{11}$ & 1.000 & $8.000^{2} \cdot 1.000=64.000$ & $4.500^{2} \cdot 1.000=20.250$ \\
\hline
\end{tabular}

Note. Parameter values in italics indicate that corresponding parameters where fixed a priori by the corresponding scaling method. Some conversions are not exact due to rounding imprecision. 
Table 7: Estimation results for the misspecified regression model.

\begin{tabular}{lrrr}
\hline Parameter & FM1 & \multicolumn{1}{c}{ EC } & \multicolumn{1}{c}{ FF } \\
\hline$\widehat{\lambda}_{x, 11}$ & 1.000 & 1.200 & 2.000 \\
$\widehat{\lambda}_{x, 21}$ & 0.500 & 0.600 & 1.000 \\
$\widehat{\lambda}_{x, 31}$ & 1.000 & 1.200 & 2.000 \\
$\widehat{\lambda}_{y, 11}$ & 1.000 & 0.641 & 4.998 \\
$\widehat{\lambda}_{y, 21}$ & 0.252 & 0.162 & 1.260 \\
$\widehat{\lambda}_{y, 31}$ & 1.246 & 0.799 & 6.225 \\
$\widehat{\lambda}_{y, 41}$ & 4.098 & 2.627 & 20.481 \\
$\widehat{\lambda}_{y, 51}$ & 0.690 & 0.442 & 3.447 \\
$\widehat{\lambda}_{y, 61}$ & 2.073 & 1.329 & 10.361 \\
$\widehat{\gamma}_{11}$ & 1.841 & 3.445 & 0.737 \\
$\widehat{\phi}_{11}$ & 4.000 & 2.778 & 1.000 \\
$\widehat{\psi}_{11}$ & 24.977 & 60.770 & 1.000 \\
$\widehat{\theta}_{\delta, 11}$ & 25.000 & 25.000 & 25.000 \\
$\widehat{\theta}_{\delta, 21}$ & 25.000 & 25.000 & 25.000 \\
$\widehat{\theta}_{\delta, 31}$ & 25.000 & 25.000 & 25.000 \\
$\widehat{\theta}_{\epsilon, 11}$ & 34.471 & 34.471 & 34.471 \\
$\widehat{\theta}_{\epsilon, 21}$ & 25.550 & 25.550 & 25.550 \\
$\widehat{\theta}_{\epsilon, 31}$ & 40.221 & 40.221 & 40.221 \\
$\widehat{\theta}_{\epsilon, 41}$ & 34.908 & 34.908 & 34.908 \\
$\widehat{\theta}_{\epsilon, 51}$ & 24.918 & 24.918 & 24.918 \\
$\widehat{\theta}_{\epsilon, 61}$ & 23.657 & 23.657 & 23.657 \\
\hline & & &
\end{tabular}




\section{Figures}

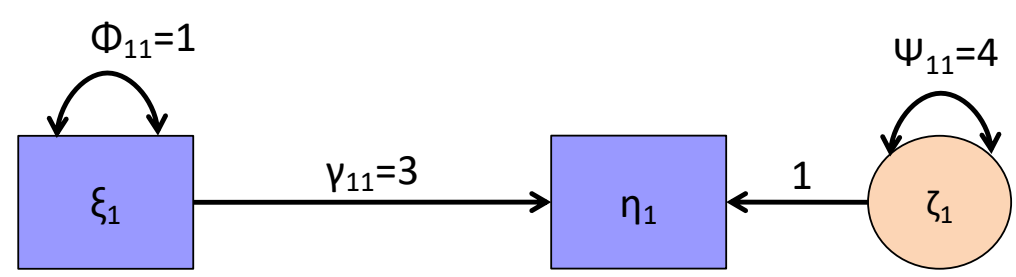

Figure 1: Population model of a manifest regression model.

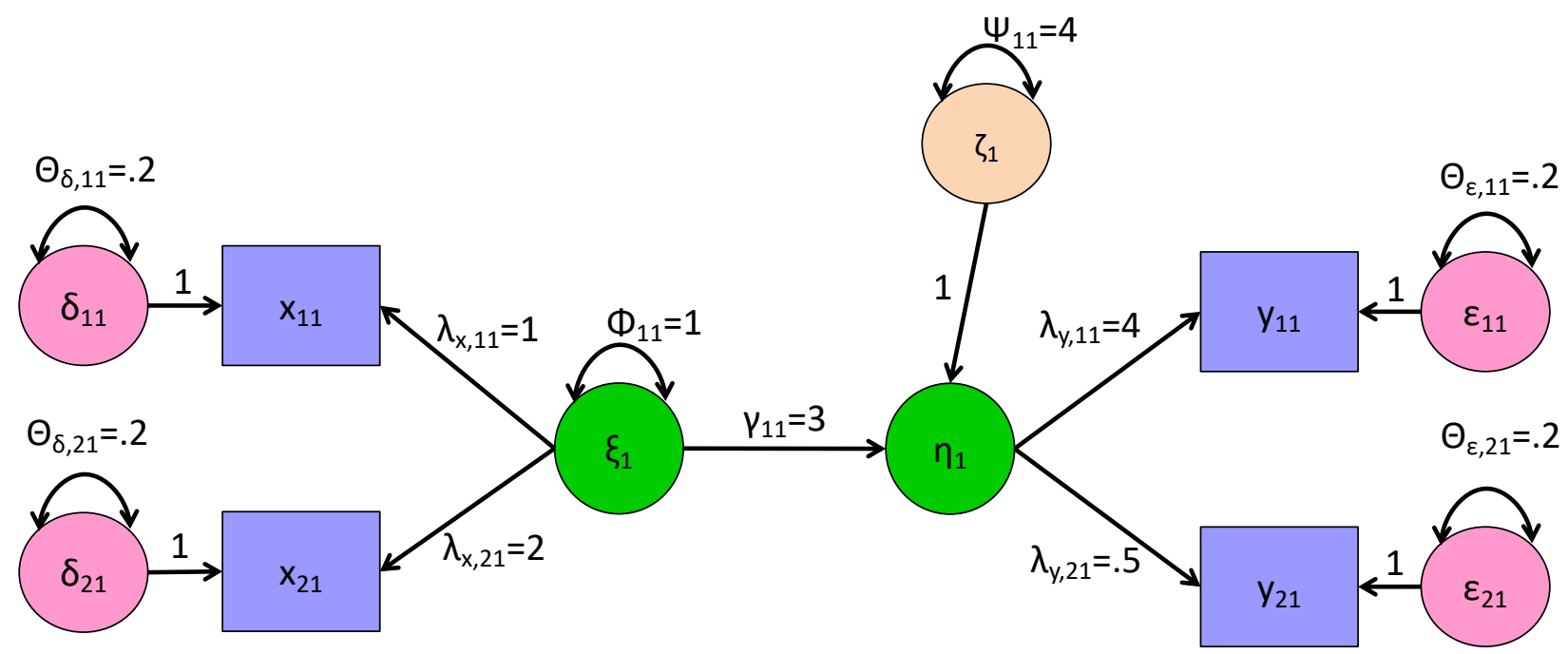

Figure 2: Population model of a regression with latent variables. 


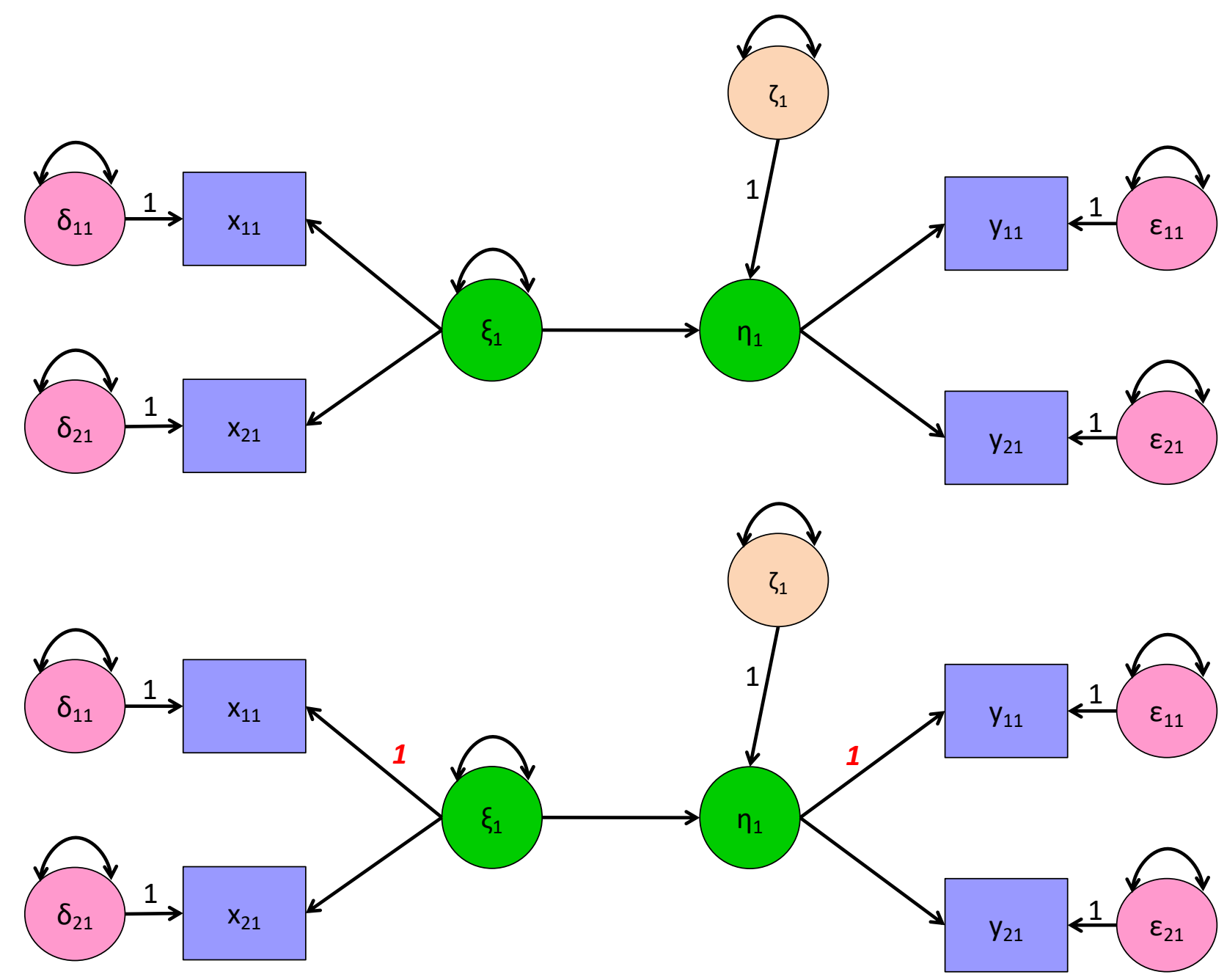

Figure 3: Example of an unscaled model (upper part) and the FM scaled model derived from it (lower part). 


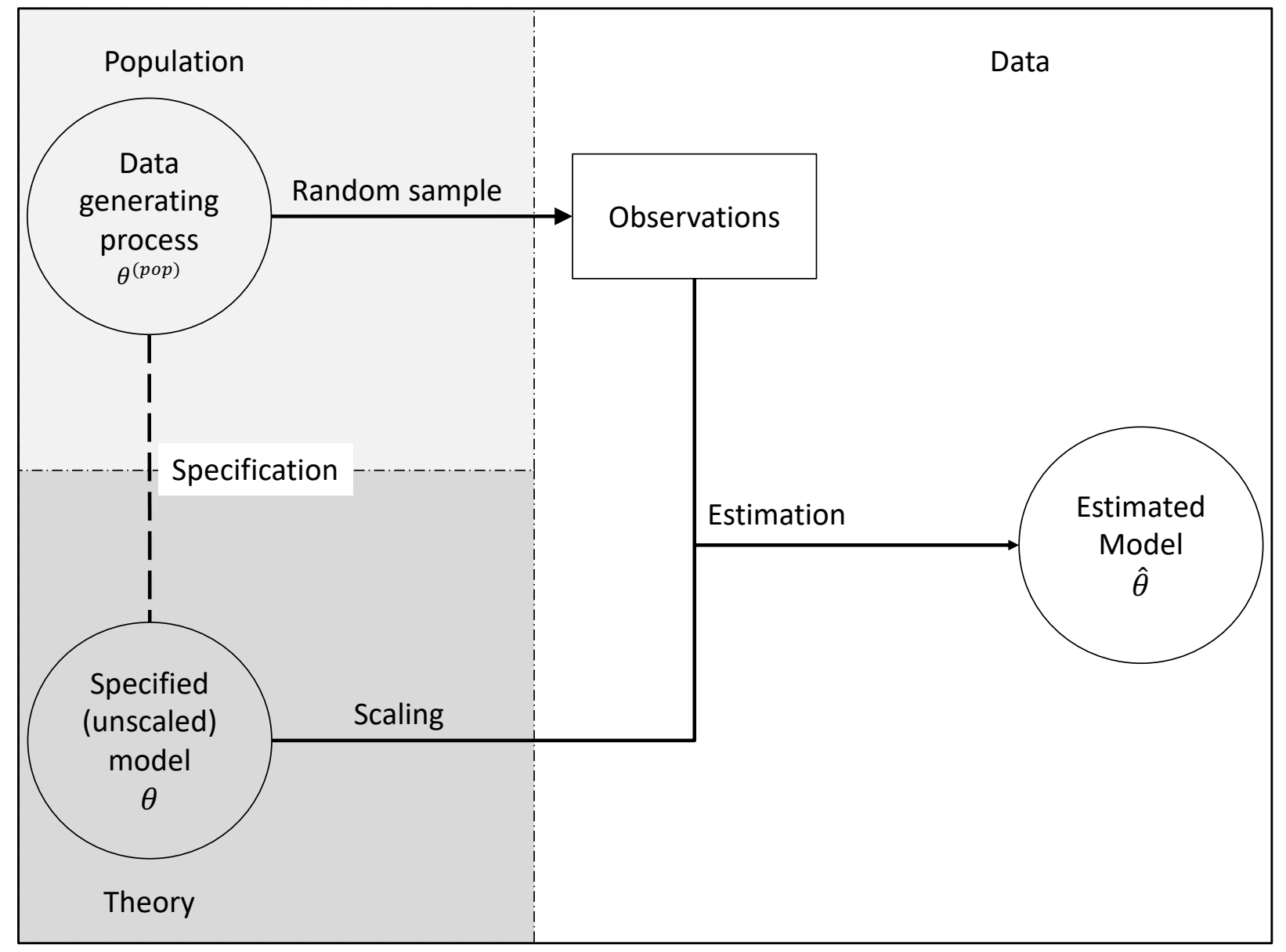

Figure 4: Relation between population, dgp, data, and specified models. 


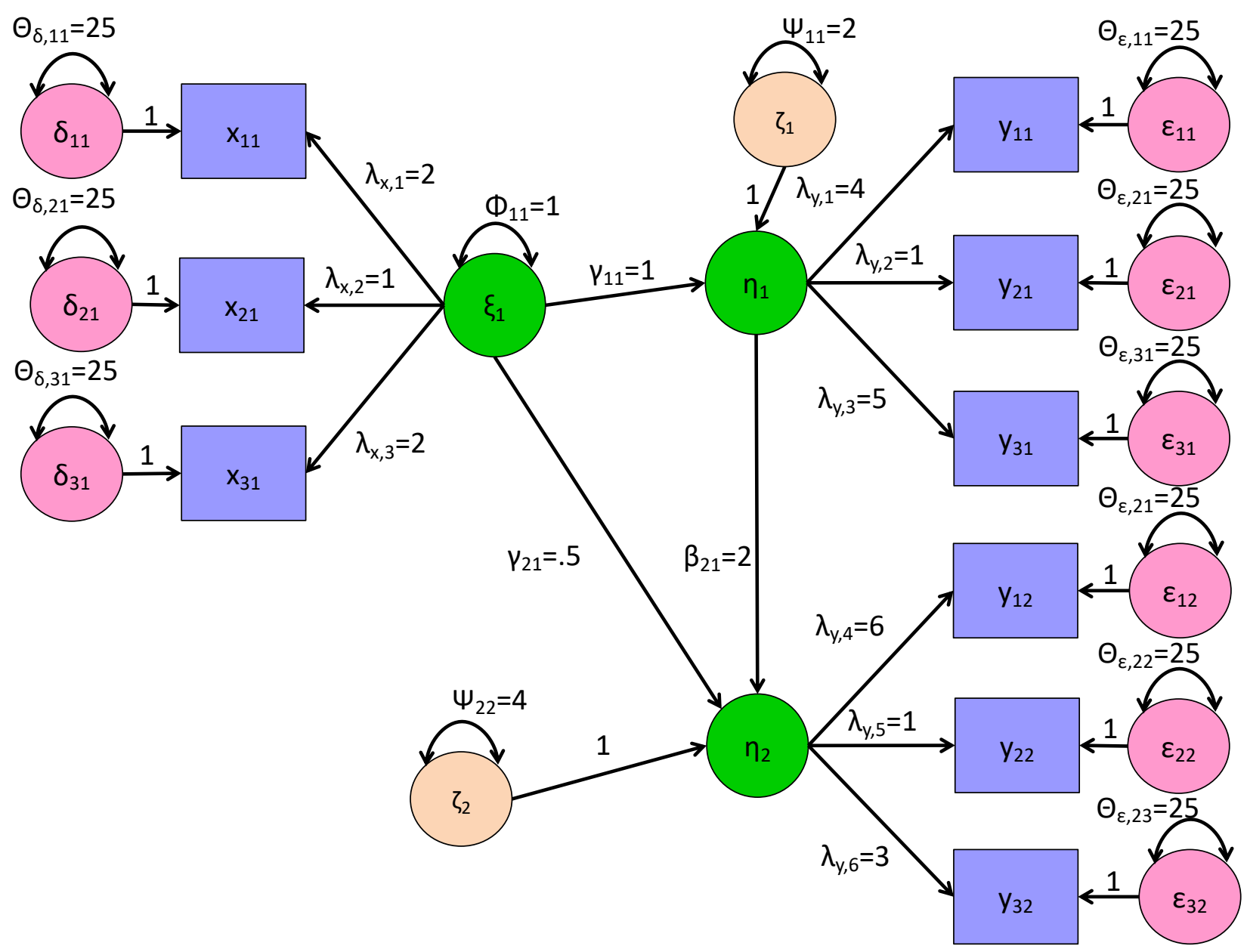

Figure 5: Population model of the mediation. 


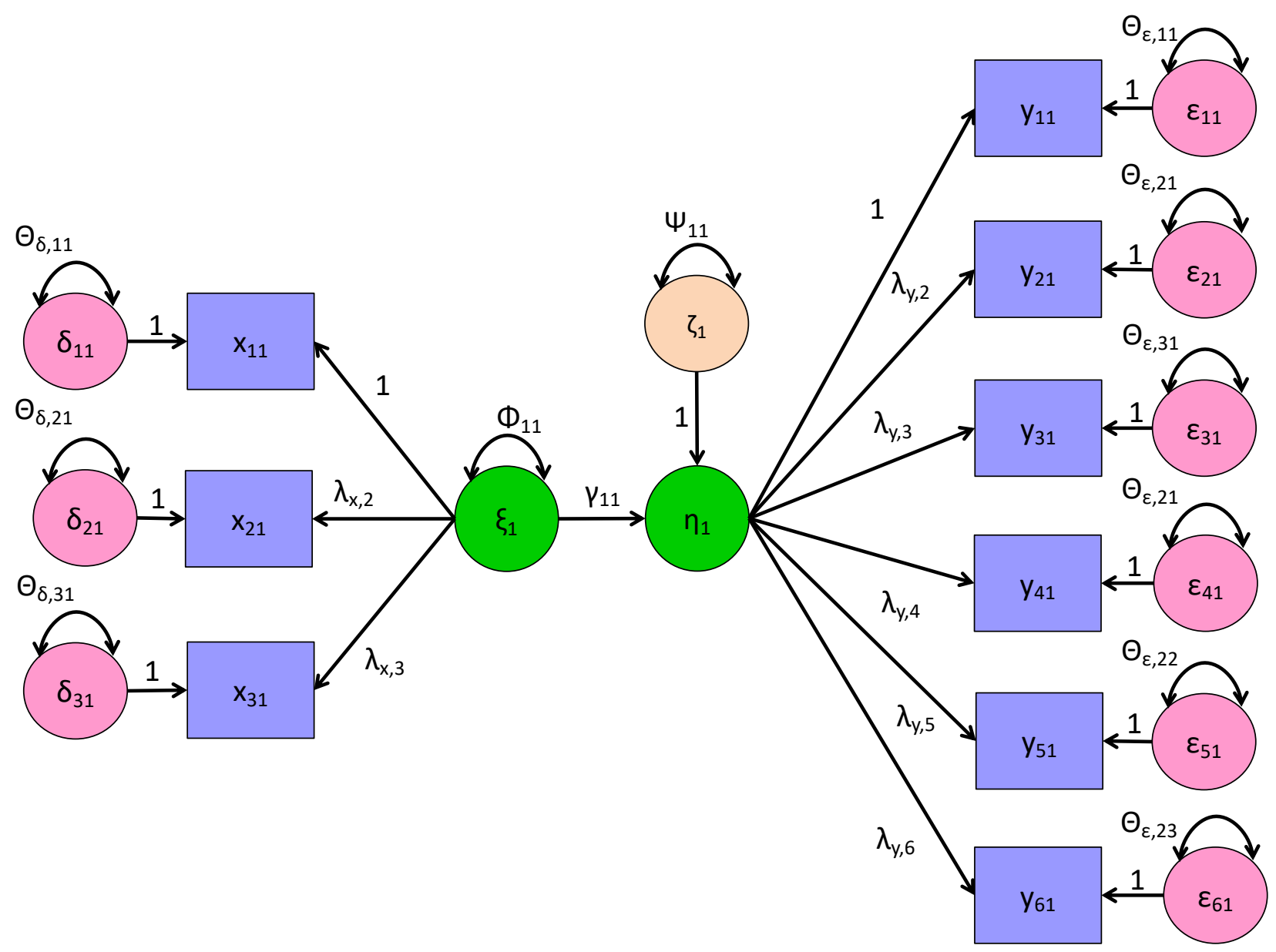

Figure 6: Regression model that is misspecified in relation to the population model in Figure 5. 

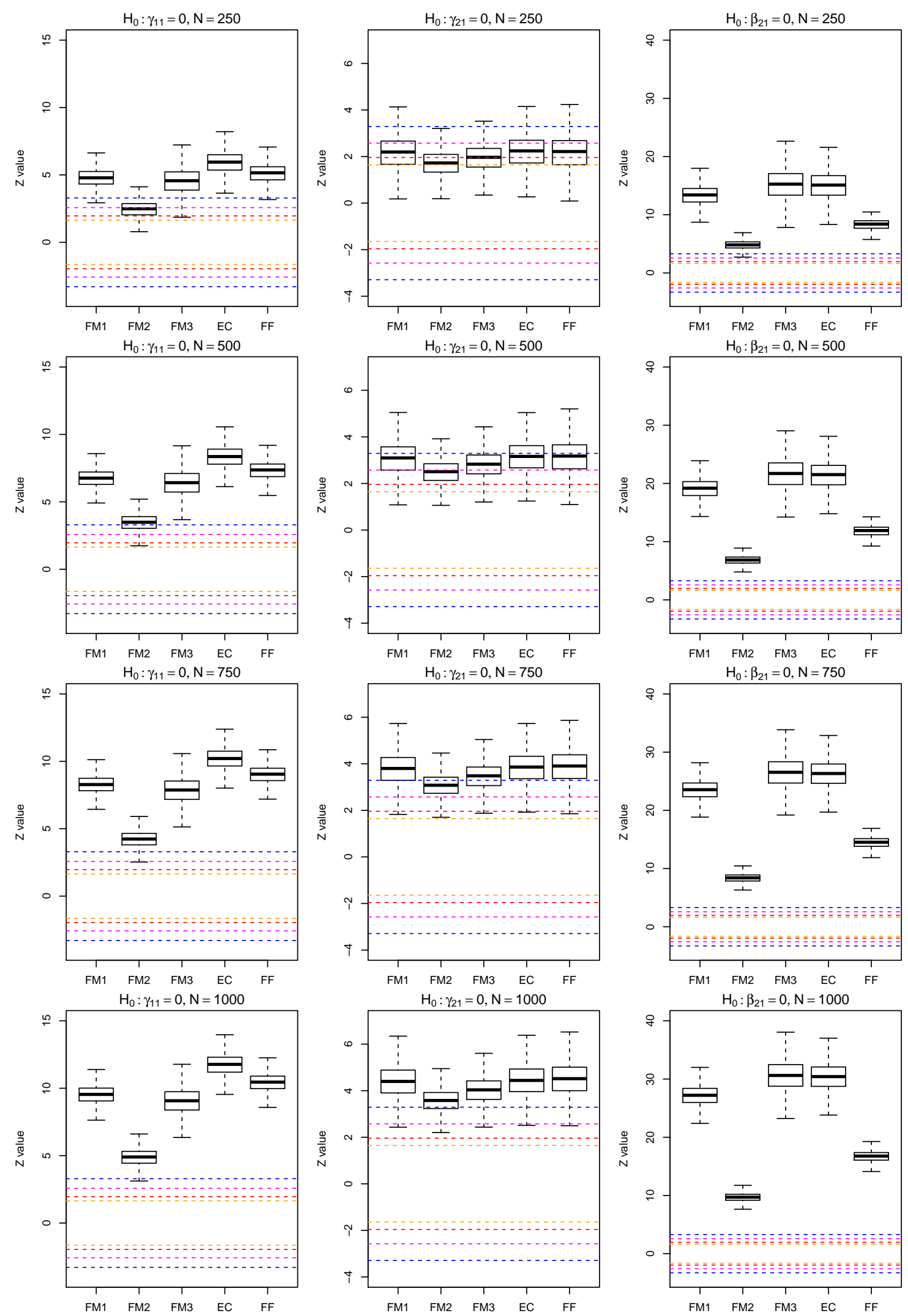

Figure 7: Results of Monte Carlo Simulation of Wald Tests under $H_{1}$. 

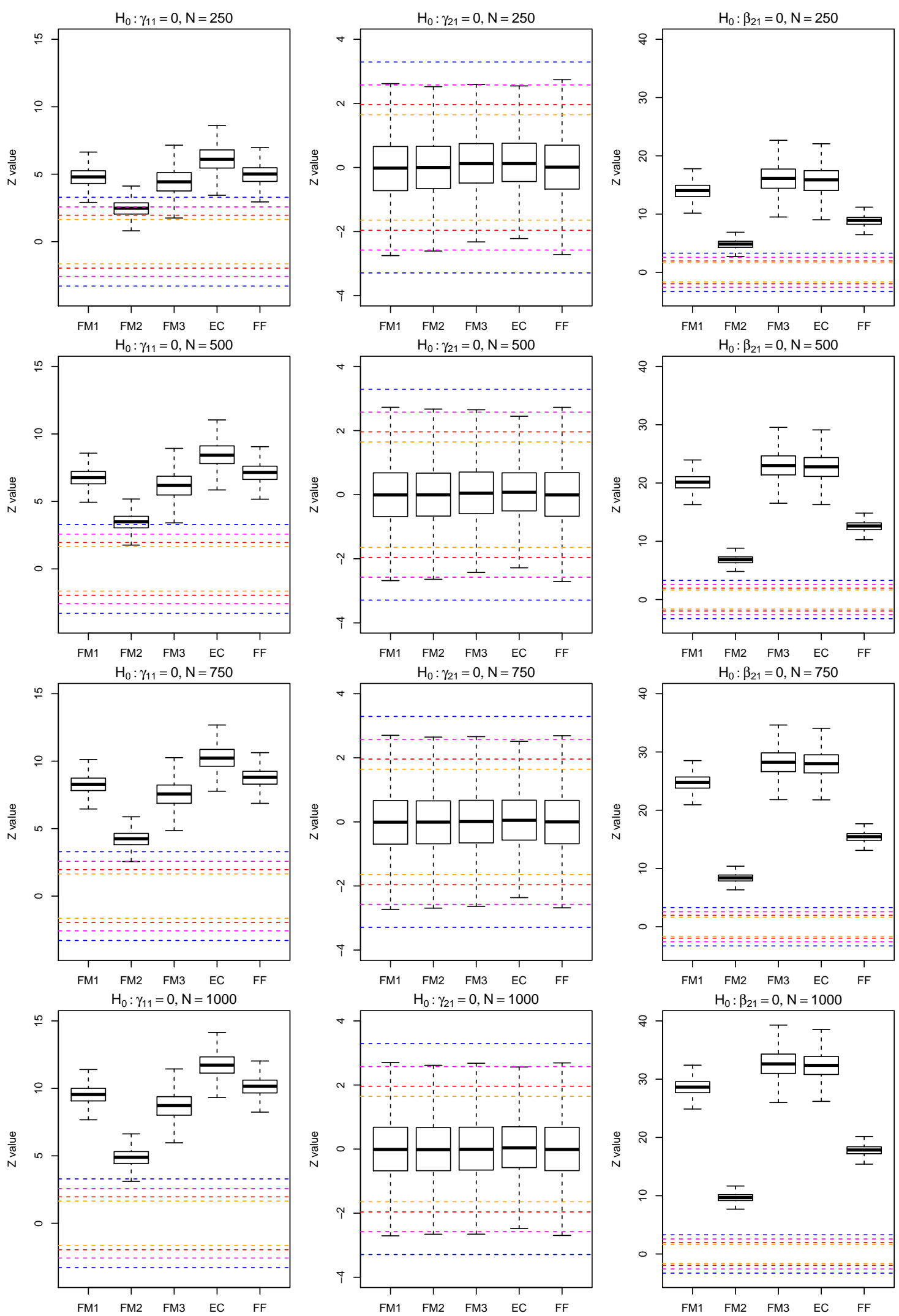

Figure 8: Results of Monte Carlo Simulation of Wald Tests under $\gamma_{21}^{(\mathrm{pop})}=0$. 